Article

\title{
A New Solution to Well-Known Hencky Problem: Improvement of In-Plane Equilibrium Equation
}

\author{
Xue Li ${ }^{1}$, Jun-Yi Sun ${ }^{1,2, * \mathbb{C}}$, Zhi-Hang Zhao ${ }^{1}$, Shou-Zhen $\mathrm{Li}^{1}$ and Xiao-Ting He ${ }^{1,2} \mathbb{D}$ \\ 1 School of Civil Engineering, Chongqing University, Chongqing 400045, China; lixuecqu@126.com (X.L.); \\ zzhcqu@163.com (Z.-H.Z.); lszcqu@163.com (S.-Z.L.); hexiaoting@cqu.edu.cn (X.-T.H.) \\ 2 Key Laboratory of New Technology for Construction of Cities in Mountain Area (Chongqing University), \\ Ministry of Education, Chongqing 400045, China \\ * Correspondence: sunjunyi@cqu.edu.cn; Tel.: +86-(0)23-65120720
}

Received: 4 April 2020; Accepted: 23 April 2020; Published: 25 April 2020

\begin{abstract}
In this paper, the well-known Hencky problem-that is, the problem of axisymmetric deformation of a peripherally fixed and initially flat circular membrane subjected to transverse uniformly distributed loads-is re-solved by simultaneously considering the improvement of the out-of-plane and in-plane equilibrium equations. In which, the so-called small rotation angle assumption of the membrane is given up when establishing the out-of-plane equilibrium equation, and the in-plane equilibrium equation is, for the first time, improved by considering the effect of the deflection on the equilibrium between the radial and circumferential stress. Furthermore, the resulting nonlinear differential equation is successfully solved by using the power series method, and a new closed-form solution of the problem is finally presented. The conducted numerical example indicates that the closed-form solution presented here has a higher computational accuracy in comparison with the existing solutions of the well-known Hencky problem, especially when the deflection of the membrane is relatively large.
\end{abstract}

Keywords: circular membrane; axisymmetric deformation; large deflection; equilibrium equation; power series method

\section{Introduction}

Membrane structures or structural components have played important roles in many fields of engineering or technology, for example, the MEMS (Micro-Electro-Mechanical Systems) devices [1], heat transfer enhancement applications [2], characterization of mechanical properties [3,4], and civil engineering [5,6]. Mathematical modelling is often necessary to study the mechanical behavior of structures $[7,8]$. However, the large deflection phenomenon of the membrane usually gives rise to some nonlinear differential equations. The boundary value problems of these somewhat intractable nonlinear equations are usually difficult to deal with analytically [9-15]. Therefore, analytical solutions for membrane problems are available in a few cases, but it is usually easy to find the numerical solutions obtained by, for example, the iterative or shooting method in the existing literature. In practice, however, analytical solutions are often found to be necessary.

Hencky, a famous German scientist, originally dealt with the problem of axisymmetric deformation of a peripherally fixed circular membrane under uniformly distributed transverse loads and presented its closed-form solution in the form of power series [16]. A computational error in [16] was corrected by Chien [17] and Alekseev [18], respectively. This problem is usually called the well-known Föppl-Hencky membrane problem, or simply the well-known Hencky problem, and its solution is called the well-known Hencky solution. This solution is the first solution for circular membrane problems and is often cited in relevant studies $[6,13,19-21]$. During the derivation of the well-known Hencky solution, however, 
the so-called small rotation angle assumption of the membrane-that is, suppose that the rotation angle $\theta$ of the membrane is so small that $\sin \theta \approx \tan \theta$-was adopted, which limits the applicability of the solution to the deflection of the membrane. Consequently, we gave up the small rotation angle assumption of the membrane and used $\sin \theta=\sqrt{1+1 / \tan ^{2} \theta}$ to establish the out-of-plane equilibrium equation, re-solved the well-known Hencky problem, and presented the closed-form solution without the small rotation angle assumption in [21]. However, the effect of the deflection on the in-plane equilibrium equation was not considered during the derivation of the solution presented in [21], and this still limits the applicability of solution to the deflection of membrane, which will be seen from the numerical example conducted in Section 3.

In this study, the well-known Hencky problem was re-solved by simultaneously considering the improvement of the out-of-plane and in-plane equilibrium equations, and a more refined closed-form solution of well-known Hencky problem was presented. The numerical example conducted indicates that the solution presented here has a higher computational accuracy in comparison with the existing solutions. The detailed derivation of the basic equations was arranged in the next section, in which the out-of-plane equilibrium equation is established under the condition of $\sin \theta=\sqrt{1+1 / \tan ^{2} \theta}$, the in-plane equilibrium equation is, for the first time, improved by considering the effect of deflection on the equilibrium between radial stress and circumferential stress, the resulting nonlinear differential equation is successfully solved by using the power series method, and finally, a new closed-form solution of the well-known Hencky problem is presented. In Section 3, a numerical example is conducted for the identification of the validity of the closed-form solution presented and the applicability of solution to the deflection of membrane. Section 4 features the concluding remarks.

\section{Membrane Equation and Its Solution}

A rotationally symmetric, linearly elastic, initially-flat circular membrane with Poisson's ratio $v$, Young's modulus of elasticity $E$, radius $a$ and thickness $h$ is peripherally clamped. The uniformly distributed transverse loads $q$ is quasi-statically applied onto the surface of the membrane, as shown in Figure 1, where $r$ is the radial coordinate in a cylindrical coordinate system $(r, \varphi, w)$ (where the polar coordinate plane $(r, \varphi)$ is located in the plane in which the geometric middle plane of the initially-flat circular unstretched membrane is located) and $w$ is the transverse coordinate of the cylindrical coordinate system as well as the transverse displacement of the membrane.

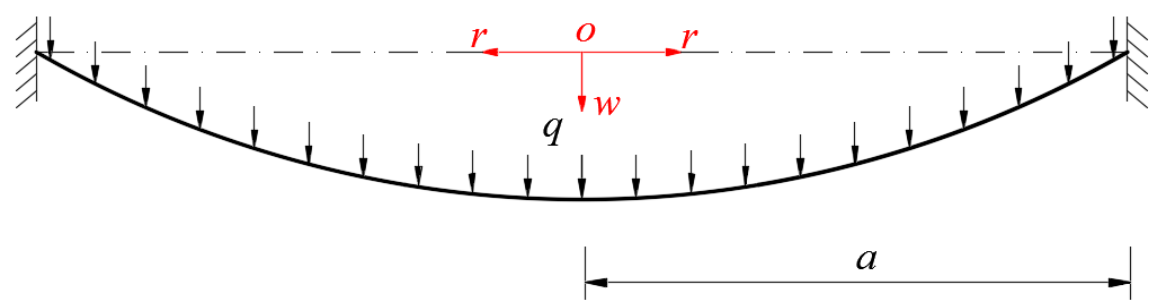

Figure 1. Sketch of the circular membrane under transverse loads $q$.

We take a piece of circular membrane with radius $0 \leq r \leq a$ in the central portion of the deformed circular membrane to study the static equilibrium problem of this piece of the deformed circular membrane under the joint actions of the external loads $q$ within $r$ and the total force $2 \pi r \sigma_{r} h$ produced by the membrane force $\sigma_{r} h$ acting on the boundary $r$, as shown in Figure 2, where $\sigma_{r}$ denotes the radial stress and $\theta$ denotes the slope angle of the deformed membrane. Clearly, there are two vertical forces, that is, the total force $\pi r^{2} q$ (in which $0 \leq r \leq a$ ) of the external loads $q$ and the total vertical membrane force $2 \pi r \sigma_{r} h \sin \theta$ that is produced by the membrane force $\sigma_{r} h$. The so-called out-of-plane equilibrium equation is

$$
2 \pi r \sigma_{r} h \sin \theta=\pi r^{2} q,
$$


where

$$
\sin \theta=1 / \sqrt{1+1 / \tan ^{2} \theta}=1 / \sqrt{1+1 /(-\mathrm{d} w / \mathrm{d} r)^{2}} .
$$

Substituting Equation (2) into Equation (1), one obtains

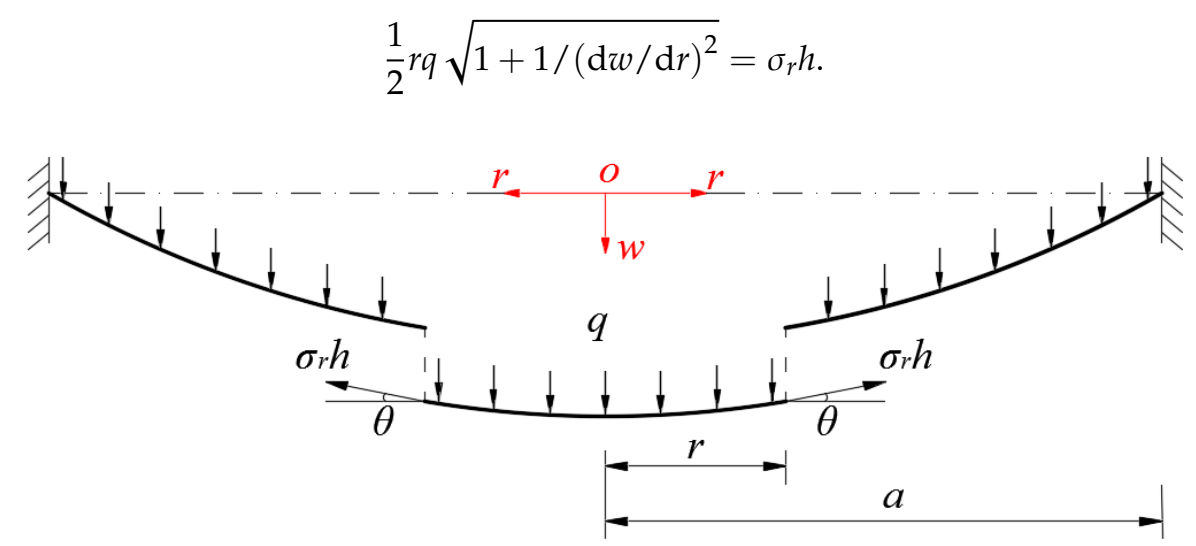

Figure 2. Sketch of the static equilibrium of the central portion $(r \leq a)$.

While in the horizontal plane which is parallel to the initially flat circular membrane, there are two horizontal forces, the circumferential membrane force $\sigma_{t} h$ and the horizontal component of the radial membrane force $\sigma_{r} h$, where $\sigma_{t}$ denotes the circumferential stress. The classic in-plane equilibrium equation, i.e., $\mathrm{d}\left(r \sigma_{r}\right) / \mathrm{d} r-\sigma_{t}=0$, is modified and replaced by

$$
\frac{\mathrm{d}}{\mathrm{d} r}\left(r \sigma_{r}\right)-\sigma_{t}\left[1+\left(-\frac{\mathrm{d} w}{\mathrm{~d} r}\right)^{2}\right]=0 .
$$

The effect of deflection on the equilibrium between radial stress and circumferential stress is thus taken into account, and for brevity, the detailed derivation of Equation (4) is arranged in Appendix A. Suppose that we denote the radial strain by $e_{r}$, circumferential strain by $e_{t}$, radial displacement by $u(r)$, and the transversal displacement by $w(r)$. The relations between the strain and displacement for the large deflection problem, that is, the so-called geometric equations, may be written as

$$
e_{r}=\frac{\mathrm{d} u}{\mathrm{~d} r}+\frac{1}{2}\left(\frac{\mathrm{d} w}{\mathrm{~d} r}\right)^{2}
$$

and

$$
e_{t}=\frac{u}{r}
$$

Moreover, the relations of the stress and strain, that is, the so-called physical equations, are still assumed to satisfy linear elasticity and can be written as

$$
\sigma_{r}=\frac{E}{1-v^{2}}\left(e_{r}+v e_{t}\right)
$$

and

$$
\sigma_{t}=\frac{E}{1-v^{2}}\left(e_{t}+v e_{r}\right) .
$$

Substituting Equations (5) and (6) into Equations (7) and (8) yields

$$
\sigma_{r}=\frac{E}{1-v^{2}}\left[\frac{\mathrm{d} u}{\mathrm{~d} r}+\frac{1}{2}\left(\frac{\mathrm{d} w}{\mathrm{~d} r}\right)^{2}+v \frac{u}{r}\right]
$$

and

$$
\sigma_{t}=\frac{E}{1-v^{2}}\left[\frac{u}{r}+v \frac{\mathrm{d} u}{\mathrm{~d} r}+\frac{v}{2}\left(\frac{\mathrm{d} w}{\mathrm{~d} r}\right)^{2}\right] .
$$


By means of Equations (9) and (10), one has

$$
\frac{u}{r}=\frac{1}{E}\left(\sigma_{t}-v \sigma_{r}\right)
$$

After substituting the $u$ of Equation (11) into Equation (9), then the so-called consistency equation can be written as

$$
(1+v)\left(\sigma_{t}-\sigma_{r}\right)+r\left(v \frac{\mathrm{d} \sigma_{r}}{\mathrm{~d} r}-\frac{\mathrm{d} \sigma_{t}}{\mathrm{~d} r}\right)+\frac{E}{2}\left(\frac{\mathrm{d} w}{\mathrm{~d} r}\right)^{2}=0 .
$$

Equations (3), (4) and (12) are three equations for the solutions of $\sigma_{r}, \sigma_{t}$ and $w(r)$. Then, the boundary conditions, under which Equations (3), (4) and (12) may be solved, are

$$
\begin{gathered}
\frac{\mathrm{d} w}{\mathrm{~d} r}=0 \text { at } r=0, \\
u=\frac{r}{E}\left(\sigma_{t}-v \sigma_{r}\right)=0 \text { at } r=a
\end{gathered}
$$

and

$$
w=0 \text { at } r=a .
$$

Let us employ the following nondimensionalization

$$
Q=\frac{a q}{h E}, W=\frac{w}{a}, S_{r}=\frac{\sigma_{r}}{E}, S_{t}=\frac{\sigma_{t}}{E}, x=\frac{r}{a},
$$

and transform Equations (3), (4) and (12)-(15) into

$$
\begin{gathered}
\left(4 S_{r}^{2}-x^{2} Q^{2}\right)\left(\frac{\mathrm{d} W}{\mathrm{~d} x}\right)^{2}-x^{2} Q^{2}=0, \\
\frac{\mathrm{d}}{\mathrm{d} x}\left(x S_{r}\right)-S_{t}\left[1+\left(\frac{\mathrm{d} W}{\mathrm{~d} x}\right)^{2}\right]=0, \\
(1+v)\left(S_{t}-S_{r}\right)+x\left(v \frac{\mathrm{d} S_{r}}{\mathrm{~d} x}-\frac{\mathrm{d} S_{t}}{\mathrm{~d} x}\right)+\frac{1}{2}\left(\frac{\mathrm{d} W}{\mathrm{~d} x}\right)^{2}=0, \\
\frac{\mathrm{d} W}{\mathrm{~d} x}=0 \text { at } x=0, \\
S_{t}-v S_{r}=0 \text { at } x=1
\end{gathered}
$$

and

$$
W=0 \text { at } x=1 .
$$

In view of the physical phenomenon that the values of stress and deflection are both finite at $x=0, S_{r}(x), W(x)$ and $S_{t}(x)$ can be expanded into the power series of the $x$, i.e., let

$$
\begin{gathered}
S_{r}(x)=\sum_{i=0}^{\infty} b_{i} x^{i}, \\
W(x)=\sum_{i=0}^{\infty} c_{i} x^{i}
\end{gathered}
$$

and

$$
S_{t}(x)=\sum_{i=0}^{\infty} d_{i} x^{i}
$$


After substituting Equations (23)-(25) into Equations (17)-(19), it is found that $b_{i} \equiv 0, c_{i} \equiv 0$ and $d_{i} \equiv 0(i=1,3,5, \ldots)$, while the coefficients $b_{i}, c_{i}$ and $d_{i}(i=2,4,6, \ldots)$ can be expressed into the polynomial with regard to the coefficients $b_{0}$ and $c_{0}$, besides $d_{0} \equiv b_{0}$ (see Appendix B).

The coefficients $b_{0}$ and $c_{0}$, as the undetermined constants depending on the concrete problem, can be determined by using the boundary conditions of Equations (21) and (22). Substituting Equations (23)-(25) into Equations (21) and (22) gives

$$
\sum_{i=0}^{\infty} d_{i}-v \sum_{i=0}^{\infty} b_{i}=0
$$

and

$$
\sum_{i=0}^{\infty} c_{i}=0 .
$$

Please note that Equations (26) and (27) contain only the undetermined constants $b_{0}$ and $c_{0}$, because $d_{0} \equiv b_{0}$ and the coefficients $b_{i}, c_{i}$ and $d_{i}(i=2,4,6, \ldots)$ were expressed into the polynomial with regard to the coefficients $b_{0}$ and $c_{0}$ at this time. Therefore, for the problem in which the values of $a, h, E, v$ and $q$ are known beforehand, the undetermined constants $b_{0}$ and $c_{0}$ can be determined by simultaneous solutions of Equations (26) and (27), and furthermore, with the known $b_{0}$ and $c_{0}$ the other coefficients $b_{i}, c_{i}, d_{i}(i=2,4,6, \ldots)$ and $d_{0}$ can be easily determined. The problem dealt with here is thus solved.

\section{Results and Discussion}

Let us firstly discuss the effectiveness of the solution obtained in Section 2. From the derivation of Section 2 of this paper and reference [21], we may see that only the in-plane equilibrium equation is modified to replace the classic in-plane equilibrium equation (i.e., $\mathrm{d}\left(r \sigma_{r}\right) / \mathrm{d} r-\sigma_{t}=0$ ). However, if we let $\mathrm{d} w / \mathrm{d} r=0$, then Equation (4) in this paper will become $\mathrm{d}\left(r \sigma_{r}\right) / \mathrm{d} r-\sigma_{t}=0$, i.e., the classic in-plane equilibrium equation which was adopted in reference [21]. This indicates that the solution presented here can regress into the solution presented in reference [21]. Furthermore, from the characteristic of axisymmetric deformation of the circular membrane, it is not difficult to understand that $\mathrm{d} w / \mathrm{d} r=0$ at $r=0$, i.e., the boundary condition Equation (13), which has not been used yet during the derivation in Section 2. Now, let us see whether the closed-form solution obtained in Section 2 meets the boundary condition of Equation (13), i.e., $\mathrm{d} w / \mathrm{d} r=0$ at $r=0$. From Equations (16) and (24) the dimensional form of the deflection $w(r)$ can be written as

$$
w(r)=\sum_{i=0}^{\infty} \frac{c_{i}}{a^{i-1}} r^{i}
$$

Then, the first derivative on both sides of Equation (28) is

$$
\frac{\mathrm{d} w}{\mathrm{~d} r}=\sum_{i=1}^{\infty} \frac{i c_{i}}{a^{i-1}} r^{i-1}
$$

Therefore, it is obvious that $\mathrm{d} w / \mathrm{d} r \equiv 0$ at $r=0$ because $\mathrm{d} w / \mathrm{d} r=c_{1}$ at $r=0$ while $c_{1} \equiv 0$. This indicates that the closed-form solution obtained in Section 2 meets the physical phenomenon of axisymmetric deformation of the circular membrane. As a result, these two aspects discussed above, to some extent, reflect the effectiveness of the closed-form solution presented here.

Let us consider a circular rubber membrane with radius $a=20 \mathrm{~mm}$, thickness $h=0.2 \mathrm{~mm}$, Young's modulus of elasticity $E=7.84 \mathrm{MPa}$, Poisson's ratio $v=0.47$ and under the action of transverse uniformly distributed loads $q$, as an example, to discuss the applicability of solution to the deflection of membrane. Figure 3 shows the variations of the deflection $w$ with the radius $r$ when $q$ takes $0.0003 \mathrm{MPa}$ and $0.03 \mathrm{MPa}$, respectively, where the solid lines represent the results obtained by the solution presented 
in Section 2, the dashed lines represent the results obtained by the solution presented in [21], and the dotted lines represent the results obtained by well-known Hencky solution [19].

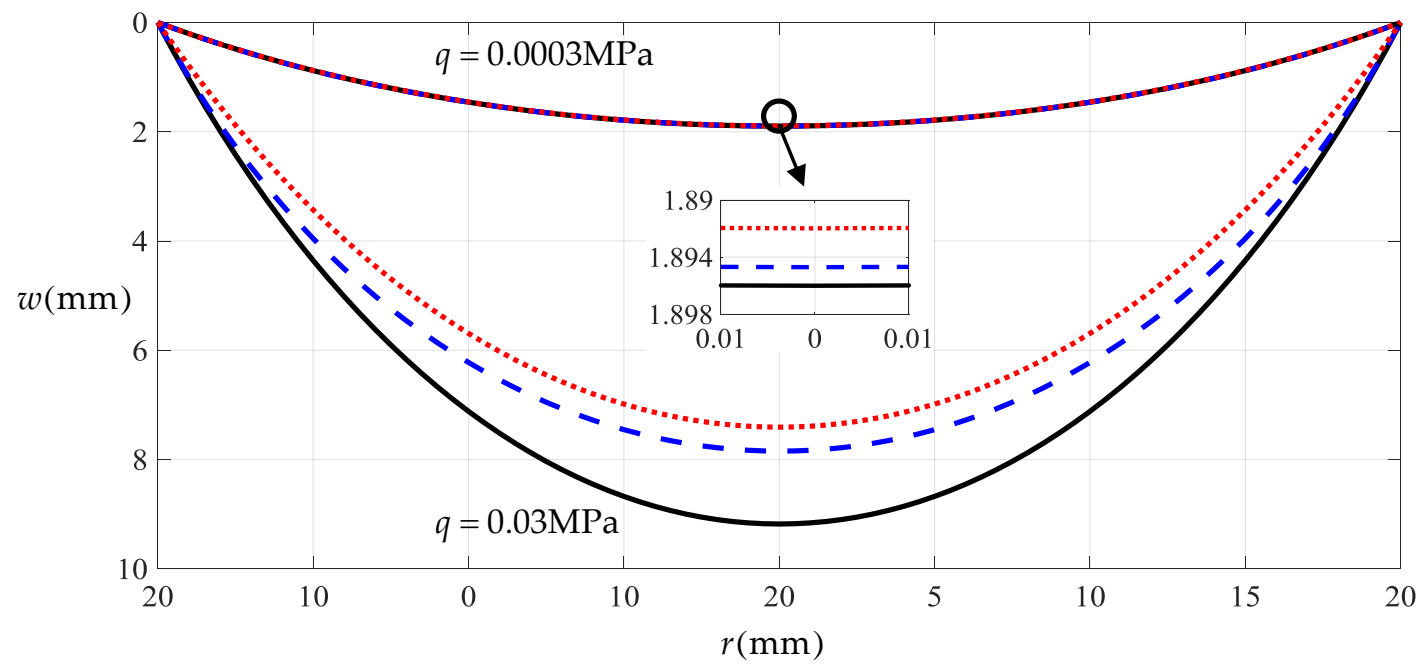

Figure 3. Variation of $w$ with $r$ when $q$ takes $0.0003 \mathrm{MPa}$ and $0.03 \mathrm{MPa}$.

From Figure 3 it may be observed that the solid line is very close to the dashed line and dotted line when $q$ takes $0.0003 \mathrm{MPa}$. This also indicates that both the closed-form solution presented here and the one presented [21] are valid, if viewed from the perspective of the well-known Hencky solution. On the other hand, along with the increase of the transverse uniformly distributed loads $q$, the deflection of the deformed membrane will also increase. Therefore, when $q$ takes $0.03 \mathrm{MPa}$, from Figure 3, we may also see that the dashed line has a certain distance from the dotted line, while the solid line has a distinct distance from the dashed line. This indicates that the small rotation angle assumption adopted in the derivation of the well-known Hencky solution limits the applicability of the solution to the deflection of the membrane, the solution after giving up the small rotation angle assumption — that is, the solution presented in [21] — can be applied to the relatively large deflection, while compared with the solution presented in [21], the solution presented in this study can be applied to the larger deflection of the membrane. When $q$ takes $0.03 \mathrm{MPa}$, the maximum deflection is about $9.18 \mathrm{~mm}, 7.85 \mathrm{~mm}$ and $7.41 \mathrm{~mm}$, respectively, which are calculated by the solution presented here, the solution presented in [21], and the well-known Hencky solution. The error between the maximum deflections calculated by the well-known Hencky solution and the one by the solution presented in [21] is about 5.94\%, which is brought by the so-called small rotation angle assumption adopted in the derivation of the well-known Hencky solution. In particular, the error between the maximum deflection calculated by the solution presented in [21] and the one by the solution presented here is about $16.94 \%$, which is brought about by the effect of the deflection ignored in the derivation of the classic in-plane equilibrium equation. It must be noted that such an error exceeds the allowable error of civil engineering, 15\%, while the allowable error of instrument design is usually less than $3 \%$, precision measurement usually less than $1 \%$, including some characterizations of mechanical properties by bulge or blister test techniques $[3-6,9,10]$.

The above discussions show that the applicability of the closed-form solution presented here to the deflection of membranes has been greatly improved, in comparison with the solution presented in [21] and the well-known Hencky solution. However, the convergence of the presented power series solution still needs to be discussed. The investigation into the convergence has to, perhaps, be arranged here, due to the fact that the coefficients $b_{i}, c_{i}, d_{i}(i=2,4,6, \ldots)$ are expressed into the somewhat intractable polynomial with regard to the coefficients $b_{0}$ and $c_{0}$ (see Appendix $\mathrm{B}$ ) and thus there is no way to discuss the convergence of the general solutions for $S_{r}(x), W(x)$ and $S_{t}(x)$. In other words, here we can prove only the convergence of the special solutions for $S_{r}(x), W(x)$ and $S_{t}(x)$, rather than 
that of its general solutions, although the convergence of the general solutions, perhaps, receives the greatest attention because the special solution will converge as long as the general solution converges.

From the derivation in Section 2, we know that the general solutions for $S_{r}(x), W(x)$ and $S_{t}(x)$ are the power series with regard to the nondimensional independent variable $x$ (see Equations (23)-(25)), where the domain of the independent variable is $0 \leq x \leq 1$ and all the coefficients $b_{i}, c_{i}$ and $d_{i}$ $(i=2,4,6, \ldots)$ are expressed into the polynomial with regard to the coefficients $b_{0}$ and $c_{0}$, besides $b_{i} \equiv 0$, $c_{i} \equiv 0, d_{i} \equiv 0(i=1,3,5, \ldots)$ and $d_{0} \equiv b_{0}$. Moreover, we also know that the undetermined constants $b_{0}$ and $c_{0}$ should be determined by simultaneous solutions of Equations (26) and (27). It seems obvious that the special solution for $S_{r}(x), W(x)$ and $S_{t}(x)$ can easily be obtained as long as the undetermined constants $b_{0}$ and $c_{0}$ can be determined. However, when solving a specific definite problem it may be found that we have to substitute the partial sum of former $n$ terms of Equations (23)-(25), rather than Equations (23)-(25), into Equations (21) and (22), otherwise the resulting Equations (26) and (27) will contain three infinite series and thus will be difficult to solve. Therefore, it seems that the undetermined constants $b_{0}$ and $c_{0}$ determined by Equations (26) and (27) will depend on the value of terms $n$ and different $n$ will determine the different value of $b_{0}$ and $c_{0}$. Hence, the discussion on convergence should contain two aspects: the variation of $b_{0}$ and $c_{0}$ with terms $n$, and the variation of $b_{i}$ and $c_{i}$ with $i$ for every value of terms $n$.

To this end, we start the numerical computation of $b_{0}$ and $c_{0}$ from $n=4$, that is, start from the partial sum of the former four terms of Equations (23)-(25), and recalculate the case of $q=0.0003 \mathrm{MPa}$ of the above numerical example. The obtained different numerical values of $b_{0}$ and $c_{0}$, are listed in Tables 1 and 2, also including the values of $b_{i}$ and $c_{i}$ corresponding to every value of $b_{0}$ and $c_{0}$. The variation of $b_{0}$ and $c_{0}$ with terms $n$ are shown in Figures 4 and 5 separately, and the variation of $b_{i}$ and $c_{i}$ with $i$ are, only for $n=30$, shown in Figures 6 and 7, respectively. From Figures 4 and 5 or Tables 1 and 2 we may see that the undetermined constants $b_{0}$ and $c_{0}$ converge reasonably well. From Figures 6 and 7 or Tables 1 and 2 we may also see that the coefficients $b_{i}$ and $c_{i}$ converge reasonably well, which indicates that the power series solutions $S_{r}(x)$ and $W(x)$ converge reasonably well because of $0 \leq x \leq 1$. Furthermore, from Figures 4 and 5 we may see that the undetermined constants $b_{0}$ and $c_{0}$ are already very close to their exact values when $n=18$. So, only the coefficients $b_{i}, c_{i}$ and $d_{i}(i=2,4,6, \ldots, 18)$, which are expressed into the polynomial with regard to $b_{0}$ and $c_{0}$, are shown in Appendix B.

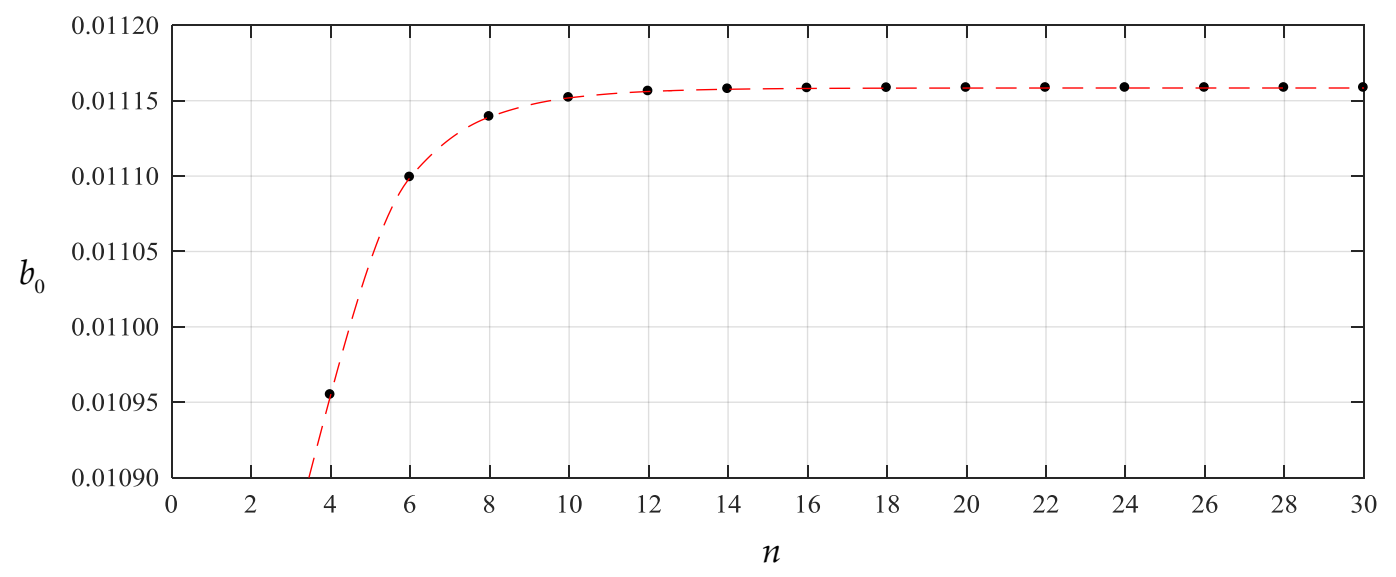

Figure 4. Variation of $b_{0}$ with $n$. 
Table 1. The values of $b_{0}-b_{6}, b_{8}-b_{14}, b_{16}-b_{22}$ and $b_{24}-b_{30}$.

\begin{tabular}{|c|c|c|c|c|}
\hline$n$ & $b_{0}$ & $b_{2}$ & $b_{4}$ & $b_{6}$ \\
\hline 4 & $1.0954809 \times 10^{-2}$ & $-1.7614908 \times 10^{-3}$ & $-2.3599430 \times 10^{-4}$ & - \\
\hline 6 & $1.1099103 \times 10^{-2}$ & $-1.7141283 \times 10^{-3}$ & $-2.2121798 \times 10^{-4}$ & $-4.7183462 \times 10^{-5}$ \\
\hline 8 & $1.1139261 \times 10^{-2}$ & $-1.7012775 \times 10^{-3}$ & $-2.1730552 \times 10^{-4}$ & $-4.5871671 \times 10^{-5}$ \\
\hline 10 & $1.1151884 \times 10^{-2}$ & $-1.6972670 \times 10^{-3}$ & $-2.1609292 \times 10^{-4}$ & $-4.5467874 \times 10^{-5}$ \\
\hline 12 & $1.1156122 \times 10^{-2}$ & $-1.6959237 \times 10^{-3}$ & $-2.1568767 \times 10^{-4}$ & $-4.5333218 \times 10^{-5}$ \\
\hline 14 & $1.1157603 \times 10^{-2}$ & $-1.6954547 \times 10^{-3}$ & $-2.1554628 \times 10^{-4}$ & $-4.5286270 \times 10^{-5}$ \\
\hline 16 & $1.1158134 \times 10^{-2}$ & $-1.6952864 \times 10^{-3}$ & $-2.1549554 \times 10^{-4}$ & $-4.5269429 \times 10^{-5}$ \\
\hline 18 & $1.1158329 \times 10^{-2}$ & $-1.6952247 \times 10^{-3}$ & $-2.1547697 \times 10^{-4}$ & $-4.5263264 \times 10^{-5}$ \\
\hline 20 & $1.1158401 \times 10^{-2}$ & $-1.6952018 \times 10^{-3}$ & $-2.1547007 \times 10^{-4}$ & $-4.5260973 \times 10^{-5}$ \\
\hline 22 & $1.1158428 \times 10^{-2}$ & $-1.6951932 \times 10^{-3}$ & $-2.1546747 \times 10^{-4}$ & $-4.5260111 \times 10^{-5}$ \\
\hline 24 & $1.1158439 \times 10^{-2}$ & $-1.6951899 \times 10^{-3}$ & $-2.1546648 \times 10^{-4}$ & $-4.5259783 \times 10^{-5}$ \\
\hline 26 & $1.1158443 \times 10^{-2}$ & $-1.6951887 \times 10^{-3}$ & $-2.1546610 \times 10^{-4}$ & $-4.5259657 \times 10^{-5}$ \\
\hline 28 & $1.1158444 \times 10^{-2}$ & $-1.6951882 \times 10^{-3}$ & $-2.1546596 \times 10^{-4}$ & $-4.5259608 \times 10^{-5}$ \\
\hline 30 & $1.1158445 \times 10^{-2}$ & $-1.6951880 \times 10^{-3}$ & $-2.1546590 \times 10^{-4}$ & $-4.5259590 \times 10^{-5}$ \\
\hline$n$ & $b_{8}$ & $b_{10}$ & $b_{12}$ & $b_{14}$ \\
\hline 8 & $-1.1940058 \times 10^{-5}$ & - & - & - \\
\hline 10 & $-1.1796657 \times 10^{-5}$ & $-3.4390107 \times 10^{-6}$ & - & - \\
\hline 12 & $-1.1748940 \times 10^{-5}$ & $-3.4213780 \times 10^{-6}$ & $-1.0729630 \times 10^{-6}$ & - \\
\hline 14 & $-1.1732316 \times 10^{-5}$ & $-3.4152393 \times 10^{-6}$ & $-1.0706309 \times 10^{-6}$ & $-3.5319449 \times 10^{-7}$ \\
\hline 16 & $-1.1726354 \times 10^{-5}$ & $-3.4130384 \times 10^{-6}$ & $-1.0697950 \times 10^{-6}$ & $-3.5287054 \times 10^{-7}$ \\
\hline 18 & $-1.1724172 \times 10^{-5}$ & $-3.4122330 \times 10^{-6}$ & $-1.0694891 \times 10^{-6}$ & $-3.5275200 \times 10^{-7}$ \\
\hline 20 & $-1.1723361 \times 10^{-5}$ & $-3.4119336 \times 10^{-6}$ & $-1.0693754 \times 10^{-6}$ & $-3.5270795 \times 10^{-7}$ \\
\hline 22 & $-1.1723055 \times 10^{-5}$ & $-3.4118209 \times 10^{-6}$ & $-1.0693326 \times 10^{-6}$ & $-3.5269137 \times 10^{-7}$ \\
\hline 24 & $-1.1722939 \times 10^{-5}$ & $-3.4117781 \times 10^{-6}$ & $-1.0693164 \times 10^{-6}$ & $-3.5268507 \times 10^{-7}$ \\
\hline 26 & $-1.1722895 \times 10^{-5}$ & $-3.4117617 \times 10^{-6}$ & $-1.0693101 \times 10^{-6}$ & $-3.5268266 \times 10^{-7}$ \\
\hline 28 & $-1.1722878 \times 10^{-5}$ & $-3.4117553 \times 10^{-6}$ & $-1.0693077 \times 10^{-6}$ & $-3.5268172 \times 10^{-7}$ \\
\hline 30 & $-1.1722871 \times 10^{-5}$ & $-3.4117529 \times 10^{-6}$ & $-1.0693068 \times 10^{-6}$ & $-3.5268136 \times 10^{-7}$ \\
\hline$n$ & $b_{16}$ & $b_{18}$ & $b_{20}$ & $b_{22}$ \\
\hline 16 & $-1.2079246 \times 10^{-7}$ & - & - & - \\
\hline 18 & $-1.2074584 \times 10^{-7}$ & $-4.2508129 \times 10^{-8}$ & - & - \\
\hline 20 & $-1.2072852 \times 10^{-7}$ & $-4.2501239 \times 10^{-8}$ & $-1.5294069 \times 10^{-8}$ & - \\
\hline 22 & $-1.2072200 \times 10^{-7}$ & $-4.2498646 \times 10^{-8}$ & $-1.5293029 \times 10^{-8}$ & $-5.6007703 \times 10^{-9}$ \\
\hline 24 & $-1.2071952 \times 10^{-7}$ & $-4.2497661 \times 10^{-8}$ & $-1.5292633 \times 10^{-8}$ & $-5.6006106 \times 10^{-9}$ \\
\hline 26 & $-1.2071857 \times 10^{-7}$ & $-4.2497283 \times 10^{-8}$ & $-1.5292482 \times 10^{-8}$ & $-5.6005494 \times 10^{-9}$ \\
\hline 28 & $-1.2071820 \times 10^{-7}$ & $-4.2497137 \times 10^{-8}$ & $-1.5292423 \times 10^{-8}$ & $-5.6005256 \times 10^{-9}$ \\
\hline 30 & $-1.2071806 \times 10^{-7}$ & $-4.2497080 \times 10^{-8}$ & $-1.5292400 \times 10^{-8}$ & $-5.6005165 \times 10^{-9}$ \\
\hline$n$ & $b_{24}$ & $b_{26}$ & $b_{28}$ & $b_{30}$ \\
\hline 24 & $-2.0808221 \times 10^{-9}$ & - & - & - \\
\hline 26 & $-2.0807972 \times 10^{-9}$ & $-7.8239857 \times 10^{-10}$ & - & - \\
\hline 28 & $-2.0807875 \times 10^{-9}$ & $-7.8239463 \times 10^{-10}$ & $-2.9717720 \times 10^{-10}$ & - \\
\hline 30 & $-2.0807839 \times 10^{-9}$ & $-7.8239312 \times 10^{-10}$ & $-2.9717659 \times 10^{-10}$ & $-1.1385721 \times 10^{-10}$ \\
\hline
\end{tabular}


Table 2. The values of $c_{0}-c_{6}, c_{8}-c_{14}, c_{16}-c_{22}$ and $c_{24}-c_{30}$.

\begin{tabular}{|c|c|c|c|c|}
\hline$n$ & $c_{0}$ & $c_{2}$ & $c_{4}$ & $c_{6}$ \\
\hline 4 & $9.5012072 \times 10^{-2}$ & $-8.7325363 \times 10^{-2}$ & $-7.6867089 \times 10^{-3}$ & - \\
\hline 6 & $9.4930058 \times 10^{-2}$ & $-8.6190091 \times 10^{-2}$ & $-7.2958154 \times 10^{-3}$ & $-1.4651512 \times 10^{-3}$ \\
\hline 8 & $9.4861056 \times 10^{-2}$ & $-8.5879367 \times 10^{-2}$ & $-7.1914770 \times 10^{-3}$ & $-1.4289971 \times 10^{-3}$ \\
\hline 10 & $9.4818023 \times 10^{-2}$ & $-8.5782156 \times 10^{-2}$ & $-7.1590654 \times 10^{-3}$ & $-1.4178443 \times 10^{-3}$ \\
\hline 12 & $9.4800124 \times 10^{-2}$ & $-8.5749571 \times 10^{-2}$ & $-7.1482256 \times 10^{-3}$ & $-1.4141226 \times 10^{-3}$ \\
\hline 14 & $9.4792979 \times 10^{-2}$ & $-8.5738190 \times 10^{-2}$ & $-7.1444425 \times 10^{-3}$ & $-1.4128247 \times 10^{-3}$ \\
\hline 16 & $9.4790167 \times 10^{-2}$ & $-8.5734105 \times 10^{-2}$ & $-7.1430850 \times 10^{-3}$ & $-1.4123591 \times 10^{-3}$ \\
\hline 18 & $9.4789066 \times 10^{-2}$ & $-8.5732609 \times 10^{-2}$ & $-7.1425880 \times 10^{-3}$ & $-1.4121887 \times 10^{-3}$ \\
\hline 20 & $9.4788634 \times 10^{-2}$ & $-8.5732053 \times 10^{-2}$ & $-7.1424033 \times 10^{-3}$ & $-1.4121253 \times 10^{-3}$ \\
\hline 22 & $9.4788465 \times 10^{-2}$ & $-8.5731844 \times 10^{-2}$ & $-7.1423338 \times 10^{-3}$ & $-1.4121015 \times 10^{-3}$ \\
\hline 24 & $9.4788399 \times 10^{-2}$ & $-8.5731765 \times 10^{-2}$ & $-7.1423074 \times 10^{-3}$ & $-1.4120924 \times 10^{-3}$ \\
\hline 26 & $9.4788373 \times 10^{-2}$ & $-8.5731734 \times 10^{-2}$ & $-7.1422972 \times 10^{-3}$ & $-1.4120890 \times 10^{-3}$ \\
\hline 28 & $9.4788362 \times 10^{-2}$ & $-8.5731722 \times 10^{-2}$ & $-7.1422933 \times 10^{-3}$ & $-1.4120876 \times 10^{-3}$ \\
\hline 30 & $9.4788358 \times 10^{-2}$ & $-8.5731718 \times 10^{-2}$ & $-7.1422918 \times 10^{-3}$ & $-1.4120871 \times 10^{-3}$ \\
\hline$n$ & $c_{8}$ & $c_{10}$ & $c_{12}$ & $c_{14}$ \\
\hline 8 & $-3.6121541 \times 10^{-4}$ & - & - & - \\
\hline 10 & $-3.5721690 \times 10^{-4}$ & $-1.0174043 \times 10^{-4}$ & - & - \\
\hline 12 & $-3.5588553 \times 10^{-4}$ & $-1.0124963 \times 10^{-4}$ & $-3.1069854 \times 10^{-5}$ & - \\
\hline 14 & $-3.5542159 \times 10^{-4}$ & $-1.0107873 \times 10^{-4}$ & $-3.1005500 \times 10^{-5}$ & $-1.0015532 \times 10^{-5}$ \\
\hline 16 & $-3.5525519 \times 10^{-4}$ & $-1.0101745 \times 10^{-4}$ & $-3.0982432 \times 10^{-5}$ & $-1.0006703 \times 10^{-5}$ \\
\hline 18 & $-3.5519429 \times 10^{-4}$ & $-1.0099502 \times 10^{-4}$ & $-3.0973990 \times 10^{-5}$ & $-1.0003472 \times 10^{-5}$ \\
\hline 20 & $-3.5517166 \times 10^{-4}$ & $-1.0098669 \times 10^{-4}$ & $-3.0970852 \times 10^{-5}$ & $-1.0002272 \times 10^{-5}$ \\
\hline 22 & $-3.5516314 \times 10^{-4}$ & $-1.0098355 \times 10^{-4}$ & $-3.0969672 \times 10^{-5}$ & $-1.0001820 \times 10^{-5}$ \\
\hline 24 & $-3.5515990 \times 10^{-4}$ & $-1.0098236 \times 10^{-4}$ & $-3.0969223 \times 10^{-5}$ & $-1.0001648 \times 10^{-5}$ \\
\hline 26 & $-3.5515866 \times 10^{-4}$ & $-1.0098190 \times 10^{-4}$ & $-3.0969051 \times 10^{-5}$ & $-1.0001582 \times 10^{-5}$ \\
\hline 28 & $-3.5515818 \times 10^{-4}$ & $-1.0098172 \times 10^{-4}$ & $-3.0968984 \times 10^{-5}$ & $-1.0001557 \times 10^{-5}$ \\
\hline 30 & $-3.5515799 \times 10^{-4}$ & $-1.0098166 \times 10^{-4}$ & $-3.0968959 \times 10^{-5}$ & $-1.0001547 \times 10^{-5}$ \\
\hline$n$ & $c_{16}$ & $c_{18}$ & $c_{20}$ & $c_{22}$ \\
\hline 16 & $-3.3561200 \times 10^{-6}$ & - & - & - \\
\hline 18 & $-3.3548675 \times 10^{-6}$ & $-1.1579367 \times 10^{-6}$ & - & - \\
\hline 20 & $-3.3544020 \times 10^{-6}$ & $-1.1577544 \times 10^{-6}$ & $-4.0875404 \times 10^{-7}$ & - \\
\hline 22 & $-3.3542269 \times 10^{-6}$ & $-1.1576858 \times 10^{-6}$ & $-4.0872695 \times 10^{-7}$ & $-1.4697409 \times 10^{-7}$ \\
\hline 24 & $-3.3541603 \times 10^{-6}$ & $-1.1576597 \times 10^{-6}$ & $-4.0871665 \times 10^{-7}$ & $-1.4696999 \times 10^{-7}$ \\
\hline 26 & $-3.3541348 \times 10^{-6}$ & $-1.1576497 \times 10^{-6}$ & $-4.0871270 \times 10^{-7}$ & $-1.4696842 \times 10^{-7}$ \\
\hline 28 & $-3.3541249 \times 10^{-6}$ & $-1.1576458 \times 10^{-6}$ & $-4.0871116 \times 10^{-7}$ & $-1.4696781 \times 10^{-7}$ \\
\hline 30 & $-3.3541211 \times 10^{-6}$ & $-1.1576444 \times 10^{-6}$ & $-4.0871058 \times 10^{-7}$ & $-1.4696758 \times 10^{-7}$ \\
\hline$n$ & $c_{24}$ & $c_{26}$ & $c_{28}$ & $c_{30}$ \\
\hline 24 & $-5.3655393 \times 10^{-8}$ & - & - & - \\
\hline 26 & $-5.3654764 \times 10^{-8}$ & $-1.9838915 \times 10^{-8}$ & - & - \\
\hline 28 & $-5.3654520 \times 10^{-8}$ & $-1.9838817 \times 10^{-8}$ & $-7.4153521 \times 10^{-9}$ & - \\
\hline 30 & $-5.3654427 \times 10^{-8}$ & $-1.9838779 \times 10^{-8}$ & $-7.4153369 \times 10^{-9}$ & $-2.7977182 \times 10^{-9}$ \\
\hline
\end{tabular}




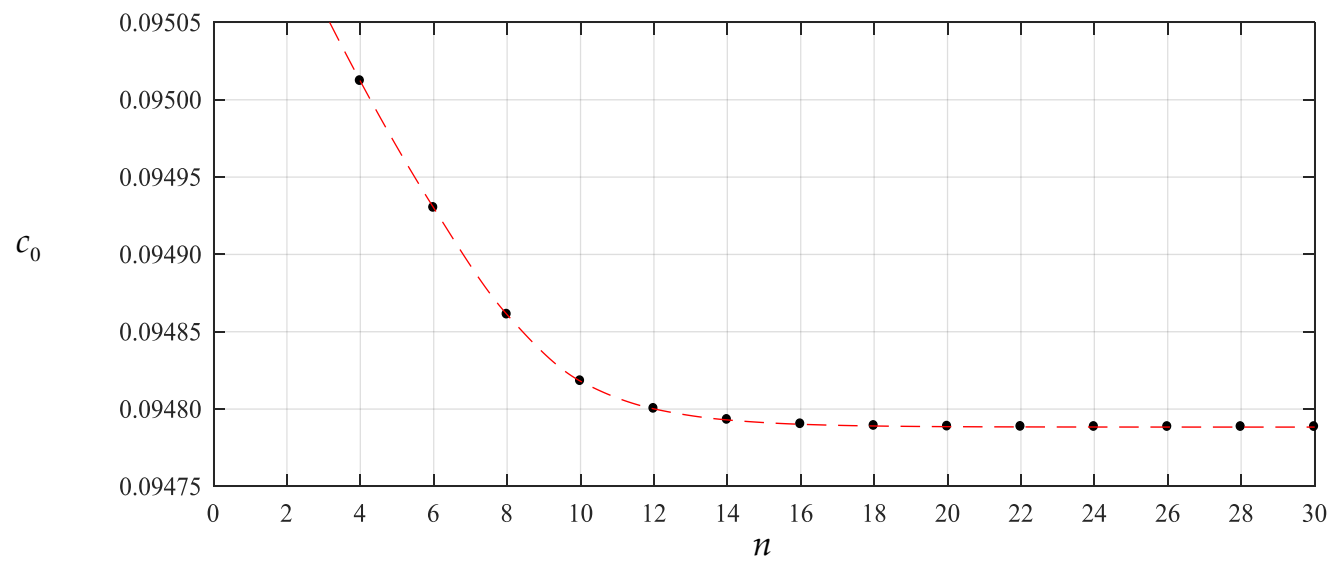

Figure 5. Variation of $c_{0}$ with $n$.

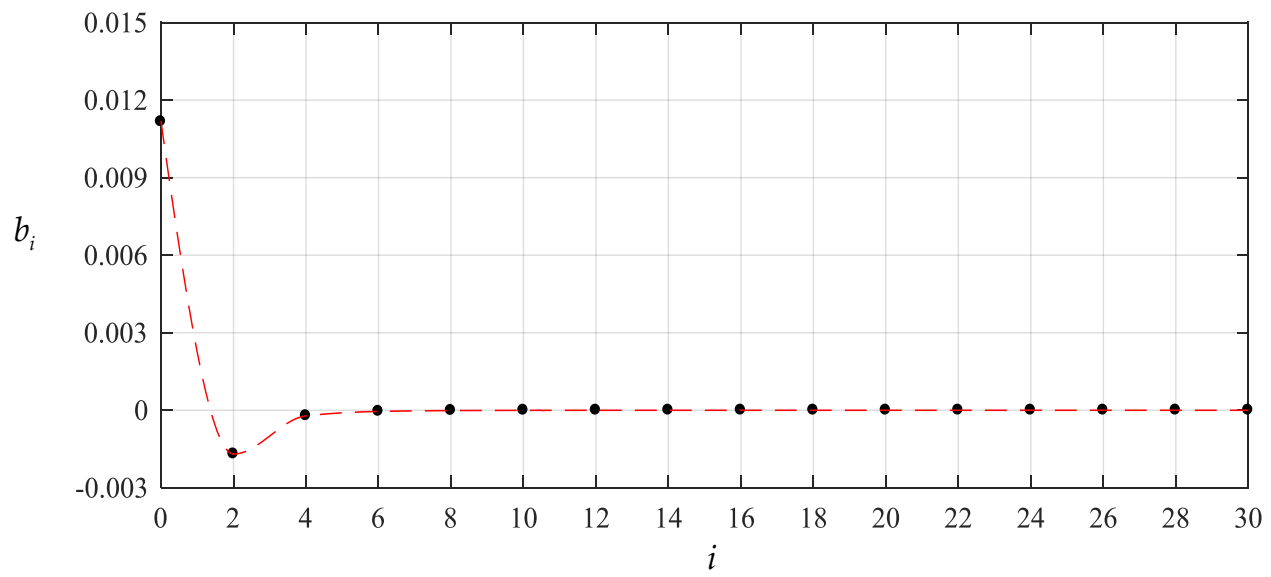

(a)

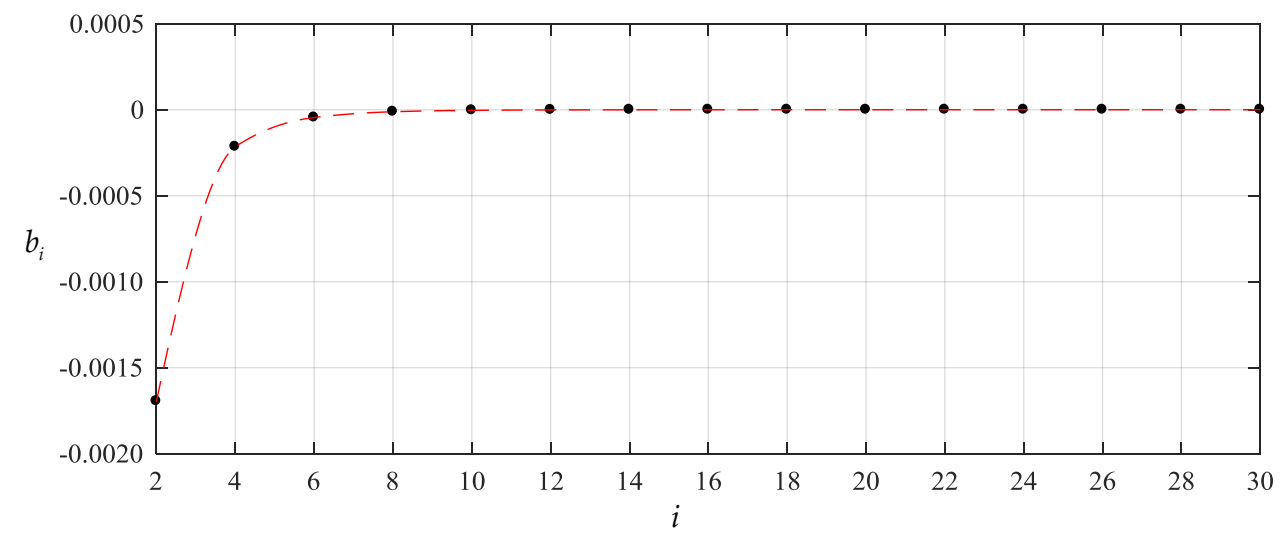

(b)

Figure 6. Variation of $b_{i}$ with $i$ for $n=30$, where (a) for $i=0,2,4,6, \ldots, 30$ and (b) for $i=2,4,6, \ldots, 30$. 


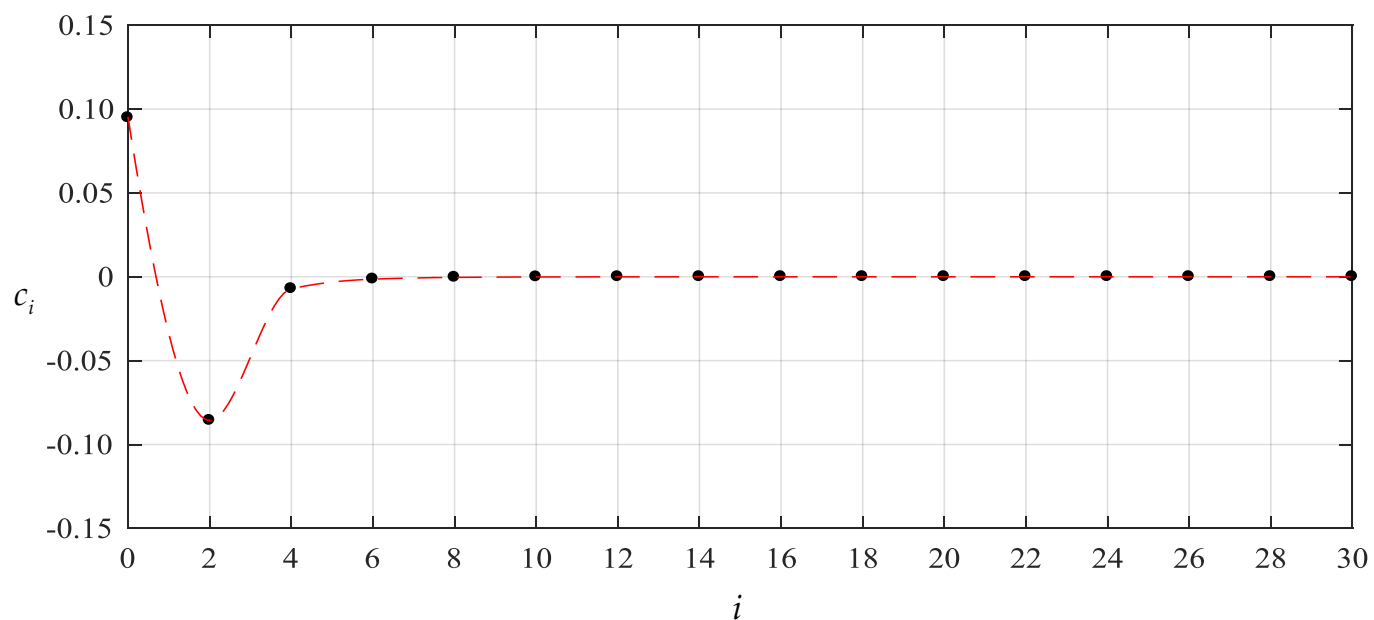

(a)

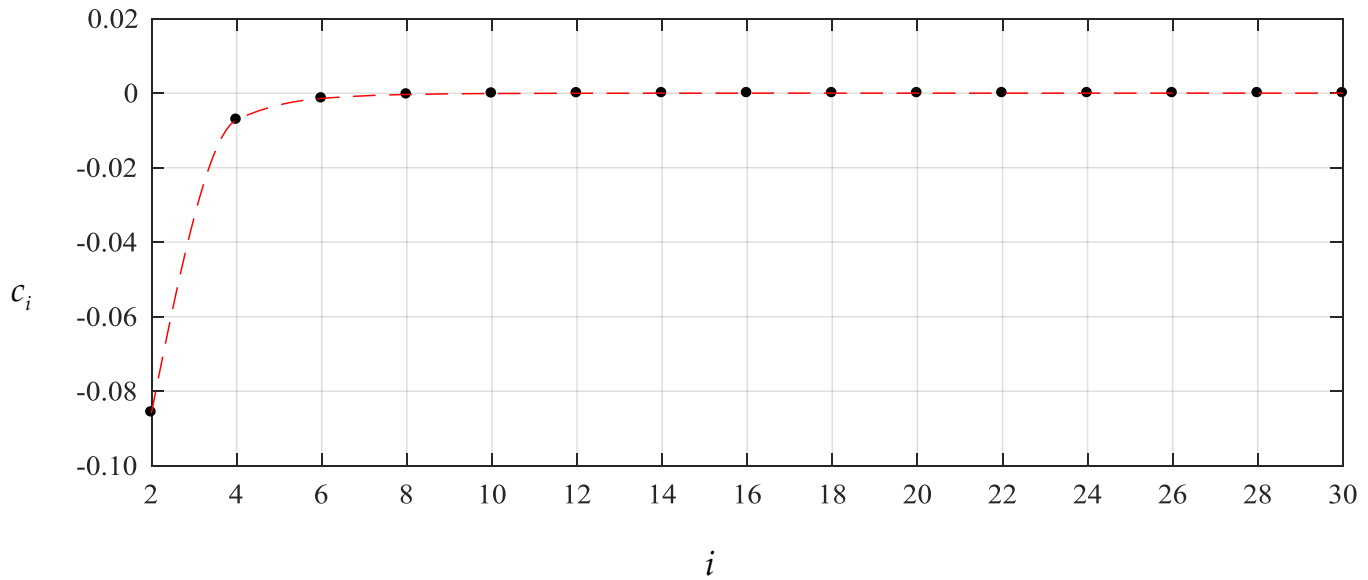

(b)

Figure 7. Variation of $c_{i}$ with $i$ for $n=30$, where (a) for $i=0,2,4,6, \ldots, 30$ and (b) for $i=2,4,6, \ldots, 30$.

\section{Concluding Remarks}

In this paper, the well-known Hencky problem was re-solved by simultaneously considering the improvement of the out-of-plane and in-plane equilibrium equations. From this study, the following conclusions can be drawn.

The well-known Hencky solution applies only to the case where the deflection of the membrane is relatively small.

Compared with the well-known Hencky solution, the solution after improving the out-of-plane equilibrium equation can be applied to the case where the deflection of the membrane is relatively large.

However, compared with the well-known Hencky solution and the solution that only the out-of-plane equilibrium equation is improved, the solution that the out-of-plane and in-plane equilibrium equations are simultaneously improved can be applied to the larger deflection of the membrane.

The large deflection phenomenon of the membrane is possible in many fields of engineering or technical application, for example, the delamination studies for the characterization of the surface and interfacial or thin-film/substrate mechanical properties by a so-called pressurized blister test, where the maximum deflection of the blistering thin film may reach half the radius of the circular blistering thin film, or even larger. Clearly, such a large deflection is not applicable to the solutions in existing 
literature. Therefore, in this sense, the work presented here should be of positive significance to these fields of technical applications.

Author Contributions: Conceptualization, X.L. and J.-Y.S.; methodology, X.L. and J.-Y.S.; validation, X.-T.H.; writing-original draft preparation, X.L. and Z.-H.Z.; writing-review and editing, X.L. and X.-T.H.; visualization, X.L. and S.-Z.L.; funding acquisition, J.-Y.S. All authors have read and agreed to the published version of the manuscript.

Funding: This research was funded by the National Natural Science Foundation of China (Grant No. 11772072).

Conflicts of Interest: The authors declare no conflict of interest.

\section{Appendix A}

Suppose that the polar coordinate $(r, \varphi)$ is set at the geometric middle-plane of the initially-flat circular membrane, and the original point of the $w$ coordinate (i.e., transverse coordinate) is set at the center point of the geometric mid-plane, thus the cylindrical coordinate system $(r, \varphi, w)$ is established, where $r$ and $\varphi$ are the radial and circumferential coordinate, respectively. We take a differential element $A B C D$ that is surrounded by two meridians $(\widehat{A B}, \widehat{C D})$ and two parallels $(\widehat{A C}, \widehat{B D})$ from the deformed membrane, to study the in-plane equilibrium of this piece under the joint actions of the radial forces $\sigma_{r} h$ and circumferential force $\sigma_{t} h$, as shown in Figure A1, where $A^{\prime} B^{\prime} C^{\prime} D^{\prime}$ is the projection of $A B C D$ on the polar coordinate plane, $\sigma_{r}$ and $\sigma_{t}$ are the radial and circumferential stress, $\Delta r$ and $\Delta \varphi$ are the radial and circumferential increment of the element $A^{\prime} B^{\prime} C^{\prime} D^{\prime}, w_{m}$ is the maximum deflection of the circular membrane, $a$ is the radius of the circular membrane, $h$ is the thickness of the deformed circular membrane.

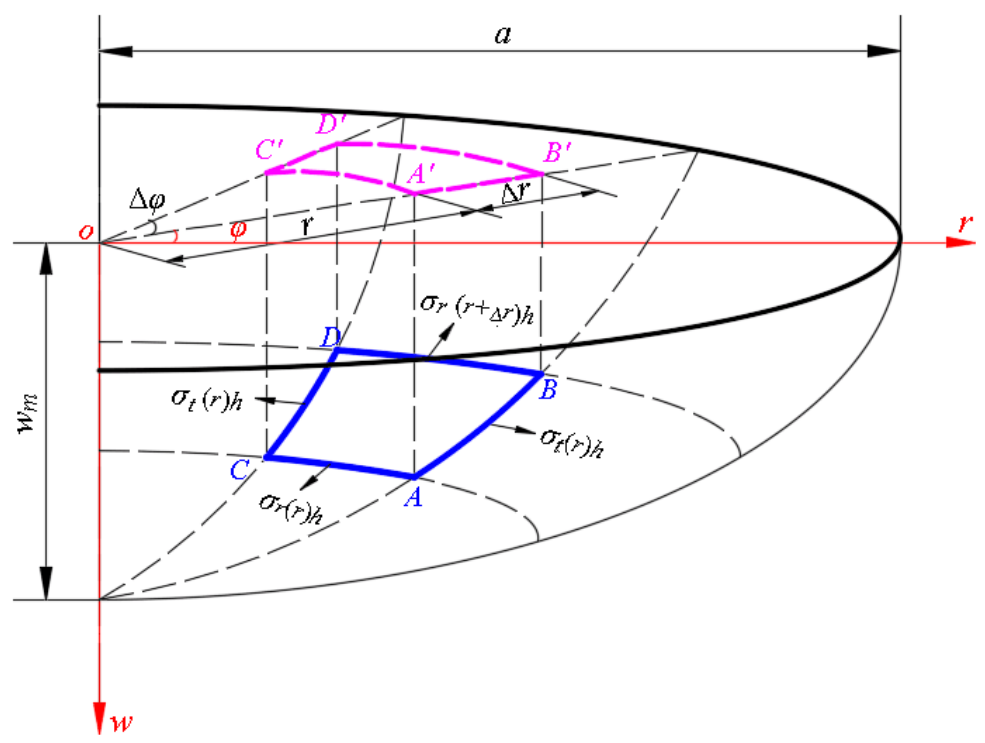

Figure A1. Diagram of the cylindrical coordinate system and the differential element $A B C D$.

In this section, the basic assumptions are made as follows: (1) the thickness of the deformed circular membrane $h$ is supposed to be constant; (2) both the radial stress and circumferential stress refer to the mean stress on the cross section of the deformed circular membrane. Subsequently, we study the static equilibrium problem of the differential element $A B C D$ under the total force in the horizontal direction, i.e., the direction parallel to the polar coordinate plane, as shown in Figure A2, where $\theta(r+\Delta r)$ and $\theta(r)$ denote the slope angles of the boundaries $r+\Delta r$ and $r$ of the differential element $A B C D, \overline{o m}$ is the angular bisector of $\angle A^{\prime} O C^{\prime}$. Clearly, there are four horizontal forces, i.e., the total force $F(r+\Delta r)$ produced by the horizontal component of radial force $\sigma_{r}(r+\Delta r) h \cos \theta(r+\Delta r)$ acting on the boundary $r+\Delta r$, the total force $F(r)$ produced by the horizontal component of radial force $\sigma_{r}(r) h \cos \theta(r)$ acting 
on the boundary $r$, two total forces $F^{\prime}$ produced by the circumferential force $\sigma_{t}(r) h$ acting on the boundaries $\widehat{A B}$ and $\widehat{C D}$. The total force $F(r+\Delta r)$ acting on the boundary $r+\Delta r$ is

$$
F(r+\Delta r)=\sigma_{r}(r+\Delta r) h \cos \theta(r+\Delta r) \cdot(r+\Delta r) \Delta \varphi,
$$

Similarly, the total force $F(r)$ acting on the boundary $r$ is

$$
F(r)=\sigma_{r}(r) h \cos \theta(r) \cdot r \Delta \varphi .
$$

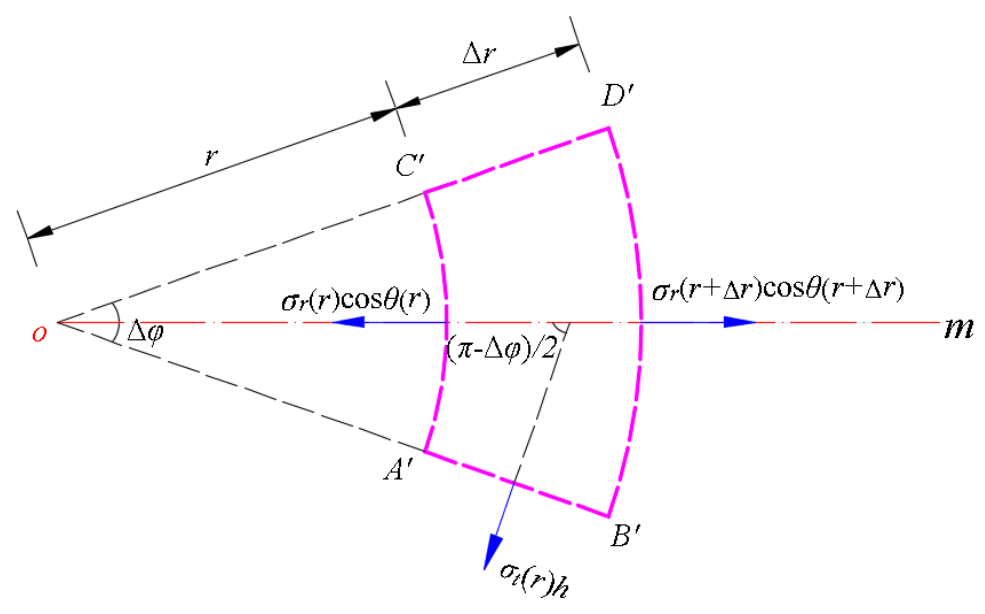

Figure A2. Diagram of the element $A^{\prime} B^{\prime} C^{\prime} D^{\prime}$ (the projection of $A B C D$ ).

Assuming that, after the membrane is deflecting, the length of the meridian $\widehat{A B}$ is approximately equal to the length of the straight line $\overline{A B}$, i.e., $\widehat{A B} \approx \overline{A B} \approx \Delta r / \cos \theta(r)$, we obtain

$$
F^{\prime}=\sigma_{t}(r) h \cdot \frac{\Delta r}{\cos \theta(r)} \cdot \cos \frac{\pi-\Delta \varphi}{2} .
$$

To sum up, the in-plane equilibrium equation can be written as

$$
F(r+\Delta r)-F(r+\Delta r)-2 F^{\prime}=0 .
$$

Due to $\Delta r \rightarrow 0$, we can assume that $\theta(r+\Delta r) \approx \theta(r)$ and record them as $\theta$. Substituting Equations (A1)-(A3) into Equation (A4), we can obtain

$$
\sigma_{r}(r+\Delta r) h(r+\Delta r) \Delta \varphi \cos \theta-\sigma_{r}(r) h r \Delta \varphi \cos \theta-2 \sigma_{t}(r) h \frac{\Delta r}{\cos \theta} \cos \frac{\pi-\Delta \varphi}{2}=0,
$$

then we expand $\sigma_{r}(r+\Delta r)$ into the Taylor series as

$$
\sigma_{r}(r+\Delta r)=\sigma_{r}(r)+\frac{\mathrm{d} \sigma_{r}(r)}{\mathrm{d} r} \Delta r+\frac{1}{2 !} \frac{\mathrm{d}^{2} \sigma_{r}(r)}{\mathrm{d} r^{2}}(\Delta r)^{2}+\ldots
$$

The second-order and higher-order differential items in Equation (A6) may be ignored, and substituting Equation (A6) into Equation (A5) yields

$$
\left[\sigma_{r} h+\frac{\mathrm{d} \sigma_{r} h}{\mathrm{~d} r} \Delta r\right](r+\Delta r) \Delta \varphi \cos \theta-\sigma_{r} h r \Delta \varphi \cos \theta-2 \sigma_{t} h \frac{\Delta r}{\cos \theta} \sin \frac{\Delta \varphi}{2}=0,
$$


after ignoring the third-order differential item and dividing the equation by $\Delta r \Delta \varphi$, we obtain

$$
\left(\sigma_{r} h+r \frac{\mathrm{d} \sigma_{r} h}{\mathrm{~d} r}\right) \cos \theta-\frac{\sigma_{t} h}{\cos \theta}=0,
$$

where

$$
\cos \theta=1 / \sqrt{1+\tan ^{2} \theta}=1 / \sqrt{1+(-\mathrm{d} w / \mathrm{d} r)^{2}} .
$$

Substituting Equation (A9) into Equation (A8) yields

$$
\frac{\mathrm{d}}{\mathrm{d} r}\left(r \sigma_{r} h\right)-\sigma_{t} h\left[1+\left(-\frac{\mathrm{d} w}{\mathrm{~d} r}\right)^{2}\right]=0 .
$$

Thus, we establish a new in-plane equilibrium equation, which is improved by considering the effect of deflection on the equilibrium between radial stress and circumferential stress.

\section{Appendix B}

$$
\begin{aligned}
& b_{2}=\frac{Q^{2}}{64 b_{0}^{2}}\left(2 v b_{0}+6 b_{0}-1\right) \\
& b_{4}=\frac{Q^{4}}{6144 b_{0}{ }^{5}}\left(2 v^{2} b_{0}^{2}+16 v b_{0}^{2}+v b_{0}+30 b_{0}^{2}-7 b_{0}-1\right) \\
& b_{6}=-\frac{Q^{6}}{4718592 b_{0}{ }^{8}}\left(48 v^{3} b_{0}^{3}+576 v^{2} b_{0}^{3}-56 v^{2} b_{0}^{2}+1968 v b_{0}^{3}-952 v b_{0}^{2}+2016 b_{0}^{3}\right. \\
& \left.-10 v b_{0}-1104 b_{0}^{2}+322 b_{0}+13\right) \\
& b_{8}=-\frac{Q^{8}}{1509949440 b_{0} 11}\left(1680 v^{3} b_{0}^{4}+704 v^{3} b_{0}^{3}+24240 v^{2} b_{0}{ }^{4}+10600 v^{2} b_{0}^{3}\right. \\
& +94320 v b_{0}{ }^{4}-574 v^{2} b_{0}^{2}-14304 v b_{0}{ }^{3}+110160 b_{0}{ }^{4}-13808 v b_{0}{ }^{2} \\
& \left.-45000 b_{0}^{3}-59 v b_{0}+414 b_{0}^{2}+4009 b_{0}+85\right) \\
& b_{10}=\frac{Q^{10}}{724775731200 b_{0}{ }^{14}}\left(5600 v^{5} b_{0}^{5}+152880 v^{4} b_{0}^{5}-1648 v^{4} b_{0}{ }^{4}+1428000 v^{3} b_{0}^{5}\right. \\
& -148008 v^{3} b_{0}{ }^{4}+5387200 v^{2} b_{0}{ }^{5}-12886 v^{3} b_{0}{ }^{3}-3113592 v^{2} b_{0}{ }^{4}+8332800 v b_{0}{ }^{5} \\
& -217146 v^{2} b_{0}{ }^{3}-7230872 v b_{0}{ }^{4}+4047120 b_{0}{ }^{5}+8567 v^{2} b_{0}{ }^{2}+1945542 v b_{0}{ }^{3} \\
& -4643160 b_{0}{ }^{4}+262408 v b_{0}{ }^{2}+2257162 b_{0}{ }^{3}+644 v b_{0}-368727 b_{0}{ }^{2}-67814 b_{0} \\
& -925) \\
& b_{12}=-\frac{Q^{12}}{974098582732800 b_{0} 17}\left(201600 v^{6} b_{0}{ }^{6}+4750560 v^{5} b_{0}{ }^{6}-9112347265280 v^{3} b_{0}{ }^{9}\right. \\
& -700096 v^{5} b_{0}^{5}+26174880 v^{4} b_{0}{ }^{6}-23198544 v^{4} b_{0}{ }^{5}-77054400 v^{3} b_{0}{ }^{6} \\
& +172124 v^{4} b_{0}{ }^{4}-266429952 v^{3} b_{0}{ }^{5}-907185600 v^{2} b_{0}{ }^{6}-15912528 v^{3} b_{0}{ }^{4} \\
& -253080416 v^{2} b_{0}{ }^{5}-2223862560 v b_{0}{ }^{6}+623486 v^{3} b_{0}{ }^{3}+403273272 v^{2} b_{0}{ }^{4} \\
& +770175168 v b_{0}{ }^{5}-1700131680 b_{0}{ }^{6}+11245950 v^{2} b_{0}{ }^{3}+370590640 v b_{0}{ }^{4} \\
& +1091214000 b_{0}{ }^{5}-355426 v^{2} b_{0}^{2}-213748110 v b_{0}{ }^{3}-144001428 b_{0}{ }^{4} \\
& -13185080 v b_{0}{ }^{2}-112129166 b_{0}{ }^{3}-22468 v b_{0}+37668090 b_{0}{ }^{2}+3091708 b_{0} \\
& +30125)
\end{aligned}
$$




$$
\begin{aligned}
& b_{14}=-\frac{Q^{14}}{6982338641028710400 b_{0}^{20}}\left(11827200 v^{7} b_{0}^{7}+1144535040 v^{6} b_{0}^{7}+147915776 v^{6} b_{0}{ }^{6}\right. \\
& +28339153920 v^{5} b_{0}^{7}+3483331584 v^{5} b_{0}{ }^{6}+279025612800 v^{4} b_{0}{ }^{7} \\
& -307693184 v^{5} b_{0}{ }^{5}+1472758272 v^{4} b_{0}{ }^{6}+1234029772800 v^{3} b_{0}{ }^{7}-11876431616 v^{4} b_{0}{ }^{5} \\
& -656063650304 v^{3} b_{0}{ }^{6}+2561630607360 v^{2} b_{0}{ }^{7}+64711744 v^{4} b_{0}{ }^{4}-152665297280 v^{3} b_{0}{ }^{5} \\
& -2487731378688 v^{2} b_{0}{ }^{6}+2255454305280 v b_{0}{ }^{7}+6778443904 v^{3} b_{0}{ }^{4}+475602146688 v^{2} b_{0}{ }^{5} \\
& -3206499678720 v b_{0}{ }^{6}+507331123200 b_{0}{ }^{7}+156866016 v^{3} b_{0}{ }^{3}+197522332864 v^{2} b_{0}{ }^{4} \\
& +1547910084096 v b_{0}{ }^{5}-1274120340480 b_{0}{ }^{6}+2974393872 v^{2} b_{0}{ }^{3}-115095078912 v b_{0}{ }^{4} \\
& +964311384960 b_{0}{ }^{5}-78610952 v^{2} b_{0}{ }^{2}-92246943872 v b_{0}{ }^{3}-304498643328 b_{0}{ }^{4}-3427569064 v b_{0}{ }^{2} \\
& \left.+3254553488 b_{0}{ }^{3}-4538390 v b_{0}+14845266576 b_{0}{ }^{2}+738329990 b_{0}+5481025\right) \\
& b_{16}=\frac{Q^{16}}{8043654114465074380800 b_{0}{ }^{23}}\left(2227097600 v^{8} b_{0}{ }^{8}+108823270400 v^{7} b_{0}{ }^{8}-2399569920 v^{7} b_{0}{ }^{7}\right. \\
& +1878512230400 v^{6} b_{0}^{8}-243147747840 v^{6} b_{0}{ }^{7}+13267527270400 v^{5} b_{0}^{8}-11390177024 v^{6} b_{0}{ }^{6} \\
& -6811784716800 v^{5} b_{0}^{7}+28109830528000 v^{4} b_{0}^{8}-259744124032 v^{5} b_{0}{ }^{6}-79310439820800 v^{4} b_{0}{ }^{7} \\
& -78699638451200 v^{3} b_{0}{ }^{8}+18079234432 v^{5} b_{0}{ }^{5}+2207463970560 v^{4} b_{0}{ }^{6}-190261226956800 v^{3} b_{0}{ }^{7} \\
& -458250126796800 v^{2} b_{0}{ }^{8}+793705014912 v^{4} b_{0}{ }^{5}+130773595767296 v^{3} b_{0}{ }^{6}-1889385331200 v^{2} b_{0}{ }^{7} \\
& -728427566131200 v b_{0}{ }^{8}-3300405248 v^{4} b_{0}{ }^{4}+10920660593344 v^{3} b_{0}{ }^{5}+272873400553984 v^{2} b_{0}{ }^{6} \\
& +437119353745920 v b_{0}{ }^{7}-384859852416000 b_{0}{ }^{8}-398122324336 v^{3} b_{0}{ }^{4}-90921607890880 v^{2} b_{0}{ }^{5} \\
& +81644790542976 v b_{0}{ }^{6}+363979673418240 b_{0}{ }^{7}-6257467656 v^{3} b_{0}{ }^{3}-13031706280848 v^{2} b_{0}{ }^{4} \\
& -143819212960320 v b_{0}{ }^{5}-100052889960960 b_{0}{ }^{6}-123995787760 v^{2} b_{0}{ }^{3}+28210788456944 v b_{0}{ }^{4} \\
& -30567607066816 b_{0}{ }^{5}+2799562406 v^{2} b_{0}{ }^{2}+5507430601544 v b_{0}{ }^{3}+25686949330192 b_{0}{ }^{4} \\
& +140586644296 v b_{0}^{2}-3180188927248 b_{0}{ }^{3}+154924777 v b_{0}-815299694478 b_{0}^{2} \\
& \left.-28068966787 b_{0}-165851725\right)
\end{aligned}
$$

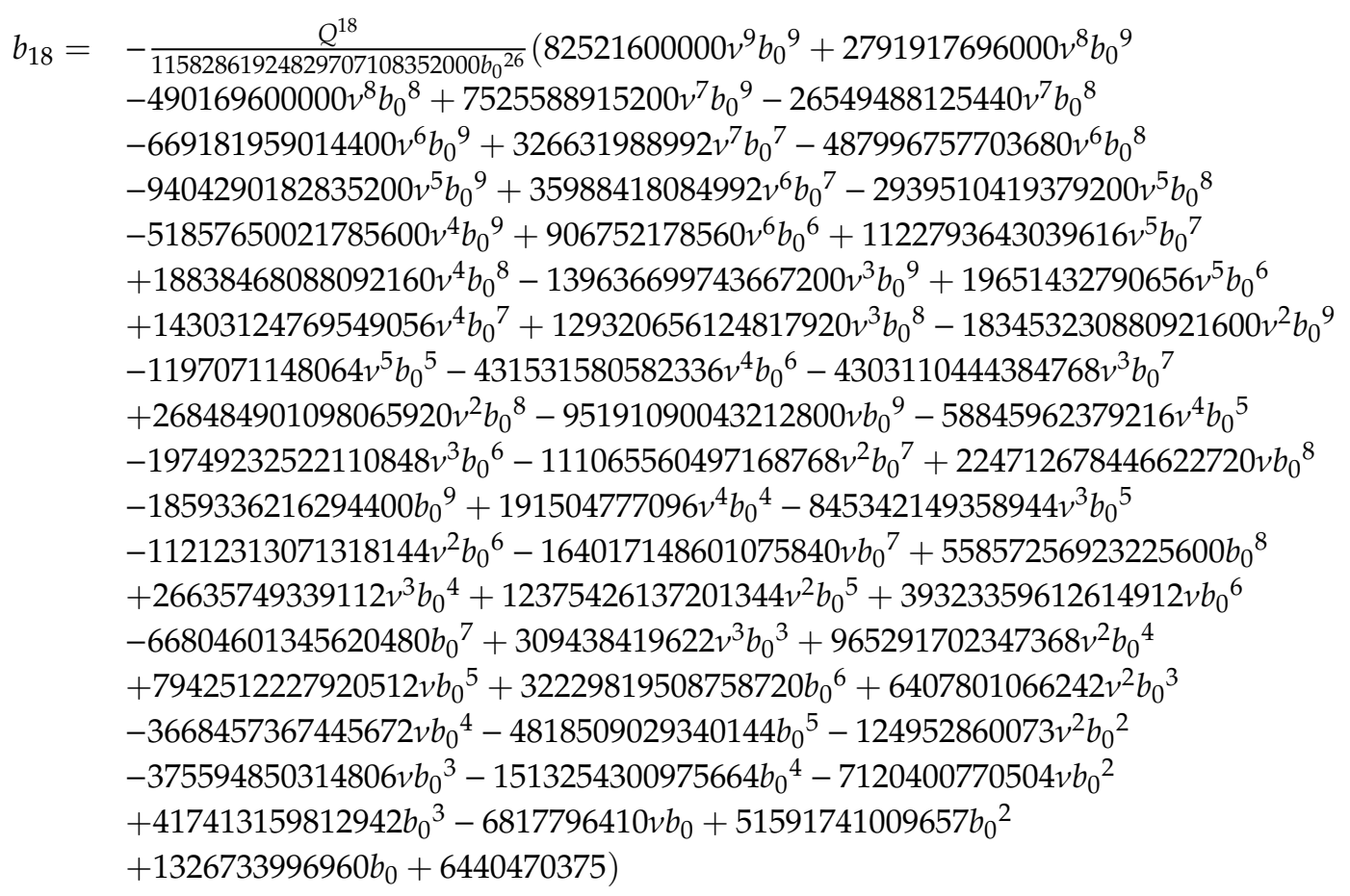

$$
\begin{gathered}
c_{2}=-\frac{1}{4} \frac{Q}{b_{0}} \\
c_{4}=\frac{Q^{3}}{512 b_{0}{ }^{4}}\left(2 v b_{0}-2 b_{0}-1\right) \\
c_{6}=-\frac{Q^{5}}{147456 b_{\mathrm{o}}{ }^{7}}\left(8 v^{2} b_{\mathrm{o}}{ }^{2}-104 v b_{\mathrm{o}}{ }^{2}-14 v b_{\mathrm{o}}-96 b_{\mathrm{o}}{ }^{2}+50 b_{\mathrm{o}}+5\right)
\end{gathered}
$$




$$
\begin{aligned}
& c_{8}=-\frac{Q^{7}}{75497472 b_{\mathrm{o}}{ }^{10}}\left(3216 v^{2} b_{\mathrm{o}}{ }^{3}+160 v^{2} b_{\mathrm{o}}{ }^{2}+672 v b_{\mathrm{o}}{ }^{3}-3976 v b_{\mathrm{o}}{ }^{2}-3888 b_{\mathrm{o}}{ }^{3}-190 v b_{\mathrm{o}}\right. \\
& \left.-600 b_{\mathrm{o}}^{2}+1174 b_{\mathrm{o}}^{2}+55\right) \\
& c_{10}=-\frac{Q^{9}}{30198988800 b_{0}{ }^{13}}\left(800 v^{4} b_{0}{ }^{4}+41840 v^{3} b_{0}{ }^{4}-304 v^{3} b_{0}{ }^{3}-204400 v^{2} b_{0}{ }^{4}\right. \\
& -109240 v^{2} b_{0}^{3}-512880 v b_{0}^{4}-2186 v^{2} b_{0}^{2}+201104 v b_{0}^{3}-246960 b_{0}{ }^{4}+81168 v b_{0}^{2} \\
& \left.+259800 b_{0}^{3}+2119 v b_{0}-48214 b_{0}^{2}-18469 b_{0}-525\right) \\
& c_{12}=-\frac{Q^{11}}{34789235097600 b_{0}{ }^{16}}\left(22400 v^{5} b_{0}^{5}+524640 v^{4} b_{0}{ }^{5}-82944 v^{4} b_{0}{ }^{4}\right. \\
& -25368000 v^{3} b_{0}^{5}-4565264 v^{3} b_{0}{ }^{4}-38892800 v^{2} b_{0}{ }^{5}+27132 v^{3} b_{0}^{3} \\
& +47745424 v^{2} b_{0}^{4}+21825600 v b_{0}{ }^{5}+7246852 v^{2} b_{0}{ }^{3}+51345424 v b_{0}{ }^{4} \\
& +41888160 b_{0}{ }^{5}+83486 v^{2} b_{0}{ }^{2}-28488284 v b_{0}{ }^{3}-7950480 b_{0}^{4}-4117416 v b_{0}{ }^{2} \\
& \left.-15997604 b_{0}{ }^{3}-70308 v b_{0}+5465194 b_{0}^{2}+784548 b_{0}+15375\right) \\
& c_{14}=-\frac{Q^{13}}{109099041266073600 b_{0}{ }^{19}}\left(537600 v^{6} b_{0}{ }^{6}+95569920 v^{5} b_{0}{ }^{6}+6967808 v^{5} b_{0}{ }^{5}+4108999680 v^{4} b_{0}{ }^{6}\right. \\
& +117889920 v^{4} b_{0}^{5}-5941056000 v^{3} b_{0}{ }^{6}-15598656 v^{4} b_{0}{ }^{4}-13529655936 v^{3} b_{0}{ }^{5}-43855603200 v^{2} b_{0}{ }^{6} \\
& -895835072 v^{3} b_{0}^{4}+4527264640 v^{2} b_{0}^{5}-51193105920 v b_{0}{ }^{6}+4503504 v^{3} b_{0}{ }^{3}+14806321024 v^{2} b_{0}{ }^{4} \\
& +46199787648 v b_{0}^{5}-14692078080 b_{0}^{6}+1109267440 v^{2} b_{0}{ }^{3}+638704192 v b_{0}{ }^{4}+26583333120 b_{0}{ }^{5} \\
& +8617304 v^{2} b_{0}^{2}-6693760592 v b_{0}^{3}-11987671488 b_{0}{ }^{4}-526821480 v b_{0}^{2}-708808304 b_{0}^{3} \\
& \left.-6494634 v b_{0}+1079360848 b_{0}^{2}+87711654 b_{0}+1278825\right) \\
& c_{16}=\frac{Q^{15}}{223434836512918732800 b_{0}^{22}}\left(171315200 v^{7} b_{0}{ }^{7}+10546508800 v^{6} b_{0}{ }^{7}-194672640 v^{6} b_{0}{ }^{6}\right. \\
& +197223398400 v^{5} b_{0}^{7}-32903308800 v^{5} b_{0}{ }^{6}-3046134348800 v^{4} b_{0}{ }^{7}-929164032 v^{5} b_{0}{ }^{5} \\
& -1387953146880 v^{4} b_{0}{ }^{6}-9506996684800 v^{3} b_{0}{ }^{7}-8465584512 v^{4} b_{0}{ }^{5}+7069457303040 v^{3} b_{0}{ }^{6} \\
& -4264563225600 v^{2} b_{0}{ }^{7}+1604448896 v^{4} b_{0}{ }^{4}+2675117507712 v^{3} b_{0}{ }^{5}+18457540600320 v^{2} b_{0}{ }^{6} \\
& +9209933798400 v b_{0}{ }^{7}+95661764736 v^{3} b_{0}{ }^{4}-5771991623808 v^{2} b_{0}{ }^{5}+6869318952960 v b_{0}{ }^{6} \\
& +7399819238400 b_{0}{ }^{7}-415417792 v^{3} b_{0}{ }^{3}-2198052940352 v^{2} b_{0}{ }^{4}-11401492164480 v b_{0}{ }^{5} \\
& -4135383590400 b_{0}^{6}-101256295408 v^{2} b_{0}{ }^{3}+1970481959168 v b_{0}{ }^{4}-2405880852480 b_{0}{ }^{5} \\
& -580531224 v^{2} b_{0}^{2}+823323522272 v b_{0}{ }^{3}+2270336257600 b_{0}{ }^{4}+42051124104 v b_{0}{ }^{2} \\
& \left.-235589110320 b_{0}^{3}+398267670 v b_{0}-115950087120 b_{0}^{2}-6276580470 b_{0}-71612125\right) \\
& c_{18}=-\frac{Q^{17}}{289571548120742677708800 b_{0}{ }^{25}}\left(5501440000 v^{8} b_{0}^{8}+152821734400 v^{7} b_{0}^{8}\right. \\
& -33579325440 v^{7} b_{0}^{7}-4940656977920 v^{6} b_{0}^{8}-2209604924928 v^{6} b_{0}{ }^{7}-300290345943040 v^{5} b_{0}{ }^{8} \\
& +23923075328 v^{6} b_{0}{ }^{6}-36967367099904 v^{5} b_{0}{ }^{7}-233731082736640 v^{4} b_{0}{ }^{8}+4138491043456 v^{5} b_{0}{ }^{6} \\
& +1140951798222336 v^{4} b_{0}{ }^{7}+2357603588480000 v^{3} b_{0}^{8}+65086315904 v^{5} b_{0}^{5} \\
& +173447529754368 v^{4} b_{0}{ }^{6}+1275723991676928 v^{3} b_{0}{ }^{7}+5390378527518720 v^{2} b_{0}{ }^{8} \\
& +35610322560 v^{4} b_{0}{ }^{5}-1573946374751744 v^{3} b_{0}{ }^{6}-3465402511093248 v^{2} b_{0}{ }^{7} \\
& +3842669495715840 v b_{0}{ }^{8}-94576563808 v^{4} b_{0}{ }^{4}-254969121731776 v^{3} b_{0}{ }^{5} \\
& -1718708981693440 v^{2} b_{0}^{6}-5807195851405824 v b_{0}{ }^{7}+634481339120640 b_{0}{ }^{8} \\
& -5837086327184 v^{3} b_{0}{ }^{4}+1016232816565696 v^{2} b_{0}{ }^{5}+1620608873701248 v b_{0}{ }^{6} \\
& -2039259064158720 b_{0}{ }^{7}+22204890216 v^{3} b_{0}{ }^{3}+173398094999376 v^{2} b_{0}{ }^{4}+879410465017152 v b_{0}{ }^{5} \\
& +1545015484836864 b_{0}{ }^{6}+5525295804496 v^{2} b_{0}{ }^{3}-312027410346224 v b_{0}{ }^{4}-238008955824704 b_{0}{ }^{5} \\
& +24785089618 v^{2} b_{0}^{2}-56402985618728 v b_{0}{ }^{3}-154810955169904 b_{0}{ }^{4}-2060926116184 v b_{0}^{2} \\
& \left.+36774798322480 b_{0}{ }^{3}-15648654025 v b_{0}+7115041992006 b_{0}{ }^{2}+280551889075 b_{0}+2596581625\right) \\
& d_{0}=b_{0} \\
& d_{2}=\frac{Q^{2}}{64 b_{\mathrm{o}}^{2}}\left(6 v b_{\mathrm{o}}+2 b_{\mathrm{o}}-3\right) \\
& d_{4}=\frac{Q^{4}}{6144 b_{\mathrm{o}}{ }^{5}}\left(10 v^{2} b_{\mathrm{o}}{ }^{2}+32 v b_{\mathrm{o}}{ }^{2}+5 v b_{\mathrm{o}}+6 b_{\mathrm{o}}{ }^{2}-11 b_{\mathrm{o}}-5\right)
\end{aligned}
$$




$$
\begin{aligned}
& d_{6}=\frac{Q^{6}}{4718592 b_{\mathrm{o}}{ }^{8}}\left(336 v^{3} b_{\mathrm{o}}{ }^{3}+1728 v^{2} b_{\mathrm{o}}{ }^{3}-392 v^{2} b_{\mathrm{o}}{ }^{2}+2256 v b_{\mathrm{o}}{ }^{3}-2632 v b_{\mathrm{o}}{ }^{2}\right. \\
& \left.+288 b_{\mathrm{o}}{ }^{3}-70 v b_{\mathrm{o}}-1392 b_{\mathrm{o}}{ }^{2}+814 b_{\mathrm{o}}+91\right) \\
& d_{8}=-\frac{Q^{8}}{1509949440 b_{0} 11}\left(15120 v^{3} b_{0}^{4}+6336 v^{3} b_{0}^{3}+83760 v^{2} b_{0}{ }^{4}+31400 v^{2} b_{0}^{3}\right. \\
& +119280 v b_{0}{ }^{4}-5166 v^{2} b_{0}{ }^{2}-35936 v b_{0}{ }^{3}+12240 b_{0}{ }^{4}-48272 v b_{0}{ }^{2}-49800 b_{0}{ }^{3} \\
& \left.-531 v b_{0}-5074 b_{0}^{2}+14081 b_{0}+765\right) \\
& d_{10}=\frac{Q^{10}}{724775731200 b_{0}{ }^{14}}\left(61600 v^{5} b_{0}^{5}+1009680 v^{4} b_{0}^{5}-18128 v^{4} b_{0}^{4}+4754400 v^{3} b_{0}^{5}\right. \\
& -1372728 v^{3} b_{0}{ }^{4}+8388800 v^{2} b_{0}{ }^{5}-141746 v^{3} b_{0}{ }^{3}-9419112 v^{2} b_{0}{ }^{4}+4771200 v b_{0}{ }^{5} \\
& -552366 v^{2} b_{0}{ }^{3}-12191752 v b_{0}{ }^{4}+367920 b_{0}{ }^{5}+94237 v^{2} b_{0}{ }^{2}+5031042 v b_{0}{ }^{3} \\
& -3194760 b_{0}{ }^{4}+1106528 v b_{0}^{2}+3840542 b_{0}^{3}+7084 v b_{0}-738837 b_{0}^{2}-304954 b_{0} \\
& -10175) \\
& d_{12}=-\frac{Q^{12}}{974098582732800 b_{0}{ }^{17}}\left(2620800 v^{6} b_{0}{ }^{6}+27888480 v^{5} b_{0}{ }^{6}-9101248 v^{5} b_{0}{ }^{5}\right. \\
& -17526240 v^{4} b_{0}^{6}-176169744 v^{4} b_{0}{ }^{5}-747691200 v^{3} b_{0}^{6}+2237612 v^{4} b_{0}^{4} \\
& -793542528 v^{3} b_{0}{ }^{5}-2150481600 v^{2} b_{0}{ }^{6}+165839280 v^{3} b_{0}{ }^{4}-125465696 v^{2} b_{0}{ }^{5} \\
& -1861138080 v b_{0}{ }^{6}+8105318 v^{3} b_{0}^{3}+1288533432 v^{2} b_{0}{ }^{4}+1580663616 v b_{0}^{5} \\
& -130779360 b_{0}{ }^{6}+19966518 v^{2} b_{0}{ }^{3}+858361168 v b_{0}{ }^{4}+841595760 b_{0}{ }^{5} \\
& -4620538 v^{2} b_{0}^{2}-656820918 v b_{0}^{3}-210868836 b_{0}{ }^{4}-65100344 v b_{0}^{2} \\
& \left.-299417606 b_{0}{ }^{3}-292084 v b_{0}+106320594 b_{0}^{2}+16945204 b_{0}+391625\right) \\
& d_{14}=-\frac{Q^{14}}{6982338641028710400 b_{0}^{20}}\left(177408000 v^{7} b_{0}{ }^{7}+14518732800 v^{6} b_{0}{ }^{7}+2218736640 v^{6} b_{0}{ }^{6}\right. \\
& +208450851840 v^{5} b_{0}{ }^{7}+17912615936 v^{5} b_{0}{ }^{6}+1086960568320 v^{4} b_{0}{ }^{7}-4615397760 v^{5} b_{0}{ }^{5} \\
& -242862506496 v^{4} b_{0}{ }^{6}+2485063680000 v^{3} b_{0}{ }^{7}-101276297472 v^{4} b_{0}{ }^{5}-2405447689728 v^{3} b_{0}{ }^{6} \\
& +2382533683200 v^{2} b_{0}{ }^{7}+970676160 v^{4} b_{0}{ }^{4}-380415886976 v^{3} b_{0}{ }^{5}-5109527995904 v^{2} b_{0}{ }^{6} \\
& +655439938560 v b_{0}{ }^{7}+79483390848 v^{3} b_{0}{ }^{4}+1497404093056 v^{2} b_{0}{ }^{5}-3515553596928 v b_{0}{ }^{6} \\
& +33822074880 b_{0}{ }^{7}+2352990240 v^{3} b_{0}{ }^{3}+676042955584 v^{2} b_{0}{ }^{4}+3056080357888 v b_{0}{ }^{5} \\
& -527848750080 b_{0}{ }^{6}+2149833968 v^{2} b_{0}{ }^{3}-209377338368 v b_{0}{ }^{4}+1136909440128 b_{0}{ }^{5} \\
& -1179164280 v^{2} b_{0}{ }^{2}-330548886912 v b_{0}{ }^{3}-554114794112 b_{0}{ }^{4}-19407979608 v b_{0}{ }^{2} \\
& \left.-36256245392 b_{0}^{3}-68075850 v b_{0}+52467583728 b_{0}^{2}+4772900250 b_{0}+82215375\right) \\
& d_{16}=\frac{Q^{16}}{8043654114465074380800 b_{0}^{23}}\left(37860659200 v^{8} b_{0}^{8}+1208591488000 v^{7} b_{0}^{8}-40792688640 v^{7} b_{0}{ }^{7}\right. \\
& +11497475891200 v^{6} b_{0}^{8}-3404657349120 v^{6} b_{0}{ }^{7}+31969385676800 v^{5} b_{0}^{8}-193633009408 v^{6} b_{0}{ }^{6} \\
& -53078925672960 v^{5} b_{0}{ }^{7}-52344910259200 v^{4} b_{0}^{8}-936859972736 v^{5} b_{0}{ }^{6}-263775135075840 v^{4} b_{0}{ }^{7} \\
& -419920548736000 v^{3} b_{0}^{8}+307346985344 v^{5} b_{0}{ }^{5}+49303741216512 v^{4} b_{0}{ }^{6}-304458419742720 v^{3} b_{0}{ }^{7} \\
& -730302465484800 v^{2} b_{0}^{8}+7485928586880 v^{4} b_{0}{ }^{5}+438011306480128 v^{3} b_{0}{ }^{6} \\
& +302399442839040 v^{2} b_{0}{ }^{7}-426376837785600 v b_{0}^{8}-56106889216 v^{4} b_{0}{ }^{4} \\
& +19774062241472 v^{3} b_{0}{ }^{5}+606307038020096 v^{2} b_{0}{ }^{6}+641582228574720 v b_{0}{ }^{7} \\
& -22638814848000 b_{0}^{8}-5212755300464 v^{3} b_{0}{ }^{4}-281654849338304 v^{2} b_{0}{ }^{5}+69515279157888 v b_{0}{ }^{6} \\
& +203356902136320 b_{0}{ }^{7}-106376950152 v^{3} b_{0}{ }^{3}-47702578054800 v^{2} b_{0}{ }^{4}-344592941602368 v b_{0}{ }^{5} \\
& -127481780298240 b_{0}{ }^{6}+65580510736 v^{2} b_{0}{ }^{3}+74542292233456 v b_{0}{ }^{4}-57032864101568 b_{0}{ }^{5} \\
& +47592560902 v^{2} b_{0}{ }^{2}+22579908784840 v b_{0}{ }^{3}+61902929849360 b_{0}{ }^{4}+898858796552 v b_{0}{ }^{2} \\
& \left.-6133979849744 b_{0}^{3}+2633721209 v b_{0}-3438453392046 b_{0}{ }^{2}-209056639379 b_{0}-2819479325\right)
\end{aligned}
$$




$$
\begin{aligned}
d_{18}= & -\frac{Q^{18}}{11582861924829707108352000 b_{0}^{26}}\left(1567910400000 v^{9} b_{0}{ }^{9}+23338660224000 v^{8} b_{0}{ }^{9}\right. \\
& -9313222400000 v^{8} b_{0}^{8}-297656437171200 v^{7} b_{0}{ }^{9}-323111917007360 v^{7} b_{0}{ }^{8} \\
& -7051459326105600 v^{6} b_{0}^{9}+6206007790848 v^{7} b_{0}^{7}-2854013744030720 v^{6} b_{0}{ }^{8} \\
& -45372968236876800 v^{5} b_{0}{ }^{9}+554595336843648 v^{6} b_{0}{ }^{7}+1536804319155200 v^{5} b_{0}{ }^{8} \\
& -132613244096102400 v^{4} b_{0}{ }^{9}+17228291392640 v^{6} b_{0}{ }^{6}+9235723372965504 v^{5} b_{0}{ }^{7} \\
& +100569211430474240 v^{4} b_{0}{ }^{8}-185303307325516800 v^{3} b_{0}{ }^{9}+21911117140864 v^{5} b_{0}{ }^{6} \\
& +40369112749918464 v^{4} b_{0}{ }^{7}+327524299398510080 v^{3} b_{0}{ }^{8}-104485247296358400 v^{2} b_{0}{ }^{9} \\
& -22744351813216 v^{5} b_{0}{ }^{5}-7530800397374784 v^{4} b_{0}{ }^{6}-47031128219899392 v^{3} b_{0}{ }^{7} \\
& +390254441572270080 v^{2} b_{0}^{8}-6864243071155200 v b_{0}{ }^{9}-607359840641904 v^{4} b_{0}{ }^{5} \\
& -64975408459152512 v^{3} b_{0}{ }^{6}-263654349683006592 v^{2} b_{0}{ }^{7}+166294391419054080 v b_{0}{ }^{8} \\
& -97859800857600 b_{0}{ }^{9}+3638590764824 v^{4} b_{0}{ }^{4}-775580213784736 v^{3} b_{0}{ }^{5} \\
& -26513210883723136 v^{2} b_{0}{ }^{6}-244351270296524160 v b_{0}{ }^{7}+12813366688934400 b_{0}{ }^{8} \\
& +386172830276728 v^{3} b_{0}{ }^{4}+40843922243052736 v^{2} b_{0}{ }^{5}+81880735502646528 v b_{0}{ }^{6} \\
& -56479479270059520 b_{0}{ }^{7}+5879329972818 v^{3} b_{0}{ }^{3}+3765115863195192 v^{2} b_{0}{ }^{4} \\
& +25412040059555328 v b_{0}{ }^{5}+47321908428655680 b_{0}{ }^{6}-12091263678602 v^{2} b_{0}{ }^{3} \\
& -11447150935542968 v b_{0}{ }^{4}-7708219666937936 b_{0}{ }^{5}-2374104341387 v^{2} b_{0}{ }^{2} \\
& -1735121877765314 v b_{0}{ }^{3}-5063597049024016 b_{0}{ }^{4}-50784882904576 v b_{0}{ }^{2} \\
& +1173843260279098 b_{0}{ }^{3}-129538131790 v b_{0}+252209221938483 b_{0}{ }^{2} \\
& \left.+11186405167240 b_{0}+122368937125\right)
\end{aligned}
$$

\section{References}

1. Di Barba, P.; Fattorusso, L.; Versaci, M. A 2D Non-linear second-order differential model for electrostatic circular membrane MEMS devices: A result of existence and uniqueness. Mathematics 2019, 7, 1193. [CrossRef]

2. Rehman, A.; Salleh, Z.; Gul, T.; Zaheer, Z. The impact of viscous dissipation on the thin film unsteady flow of GO-EG/GO-W nanofluids. Mathematics 2019, 7, 653. [CrossRef]

3. Sun, J.Y.; Lian, Y.S.; Li, Z.L.; He, X.T.; Zheng, Z.L. Theoretical study on shaft-loaded blister test technique: Synchronous characterization of surface and interfacial mechanical properties. Int. J. Adhes. Adhes. 2014, 51, 128-139. [CrossRef]

4. Sun, J.Y.; Hu, J.L.; Zheng, Z.L.; He, X.T.; Geng, H.H. A practical method for simultaneous determination of Poisson's ratio and Young's modulus of elasticity of thin films. J. Mech. Sci. Technol. 2011, 25, 3165-3171. [CrossRef]

5. Suo, H.; Angelotti, A.; Zanelli, A. Thermal-physical behavior and energy performance of air-supported membranes for sports halls: A comparison among traditional and advanced building envelopes. Energy Build. 2015, 109, 35-46. [CrossRef]

6. Król, B.; Pielichowska, K.; Król, P.; Kędzierski, M. Polyurethane cationomer films as ecological membranes for building industry. Prog. Org. Coat. 2019, 130, 83-92. [CrossRef]

7. Peride, N.; Carabineanu, A.; Craciun, E.M. Mathematical modelling of the interface crack propagation in a pre-stressed fiber reinforced elastic composite. Comp. Mater. Sci. 2009, 45, 684-692. [CrossRef]

8. Sadowski, T.; Marsavina, L.; Craciun, E.M.; Kneć, M. Modelling and experimental study of parallel cracks propagation in an orthotropic elastic material. Comp. Mater. Sci. 2012, 52, 231-235. [CrossRef]

9. Zhao, M.H.; Zheng, W.L.; Fan, C.Y. Mechanics of shaft-loaded blister test for thin film suspended on compliant substrate. Int. J. Solids Struct. 2010, 47, 2525-2532. [CrossRef]

10. Sun, J.Y.; Hu, J.L.; He, X.T.; Zheng, Z.L.; Geng, H.H. A theoretical study of thin film delamination using clamped punch-loaded blister test: Energy release rate and closed-form solution. J. Adhes. Sci. Technol. 2011, 25, 2063-2080. [CrossRef]

11. Lian, Y.S.; Sun, J.Y.; Ge, X.M.; Yang, Z.X.; He, X.T.; Zheng, Z.L. A theoretical study of an improved capacitive pressure sensor: Closed-form solution of uniformly loaded annular membranes. Measurement 2017, 111, 84-92. [CrossRef]

12. Arthurs, A.M.; Clegg, J. On the solution of a boundary value problem for the nonlinear Föppl-Hencky equation. Z. Angew. Math. Mech. 1994, 74, 281-284. [CrossRef] 
13. Lian, Y.S.; Sun, J.Y.; Dong, J.; Zheng, Z.L.; Yang, Z.X. Closed-form solution of axisymmetric deformation of prestressed Föppl-Hencky membrane under constrained deflecting. Struct. Eng. Mech. 2019, 69, 693-698.

14. Cabada, A.; Wanassi, O.K. Existence results for nonlinear fractional problems with non-homogeneous integral boundary conditions. Mathematics 2020, 8, 255. [CrossRef]

15. Jeong, J.; Kim, C.G. Existence of positive solutions to singular $\varphi$-Laplacian nonlocal boundary value problems when $\varphi$ is a sup-multiplicative-like function. Mathematics 2020, 8, 420. [CrossRef]

16. Hencky, H. Über den Spannungszustand in kreisrunden Platten mit verschwindender Biegungssteifigkeit. Z. Angew. Math. Phys. 1915, 63, 311-317.

17. Chien, W.Z. Asymptotic behavior of a thin clamped circular plate under uniform normal pressure at very large deflection. Sci. Rep. Natl. Tsinghua Univ. 1948, 5, 193-208.

18. Alekseev, S.A. Elastic circular membranes under the uniformly distributed loads. Eng. Corpus. 1953, 14, 196-198. (In Russian)

19. Sun, J.Y.; Rong, Y.; He, X.T.; Gao, X.W.; Zheng, Z.L. Power series solution of circular membrane under uniformly distributed loads: Investigation into Hencky transformation. Stuct. Eng. Mech. 2013, 45, 631-641. [CrossRef]

20. Sun, J.Y.; Lian, Y.S.; Li, Y.M.; He, X.T.; Zheng, Z.L. Closed-form solution of elastic circular membrane with initial stress under uniformly-distributed loads: Extended Hencky solution. Z. Angew. Math. Mech. 2015, 95, 1335-1341. [CrossRef]

21. Lian, Y.S.; Sun, J.Y.; Yang, Z.X.; He, X.T.; Zheng, Z.L. Closed-form solution of well-known Hencky problem without small-rotation-angle assumption. J. Appl. Math. Mech./Z. Angew. Math. Mech. 2016, 96, 1434-1441. [CrossRef]

(C) 2020 by the authors. Licensee MDPI, Basel, Switzerland. This article is an open access article distributed under the terms and conditions of the Creative Commons Attribution (CC BY) license (http://creativecommons.org/licenses/by/4.0/). 